\title{
Lattice isomorphisms between projection lattices of von Neumann algebras
}

\author{
Michiya Mori \\ Graduate School of Mathematical Sciences, The University of Tokyo, 3-8-1 Komaba, Tokyo, 153-8914, Japan; \\ E-mail: mmori@ms.u-tokyo.ac.jp.
}

Received: 17 June 2020; Revised: 18 October 2020; Accepted: 16 October 2020

2020 Mathematics Subject Classification: Primary - 46L10; Secondary - 16E50, 47B49, 51D25

\begin{abstract}
Generalizing von Neumann's result on type II $_{1}$ von Neumann algebras, I characterise lattice isomorphisms between projection lattices of arbitrary von Neumann algebras by means of ring isomorphisms between the algebras of locally measurable operators. Moreover, I give a complete description of ring isomorphisms of locally measurable operator algebras when the von Neumann algebras are without type II direct summands.
\end{abstract}

\section{Contents}

1 Introduction $\quad 1$

2 Preliminaries $\quad 3$

2.1 Various isomorphisms of von Neumann algebras . . . . . . . . . . . . 3

2.2 The algebra of locally measurable operators . . . . . . . . . . . . . . . . . 4

2.3 Halmos's two-projection theorem . . . . . . . . . . . . . . . . . . . 5

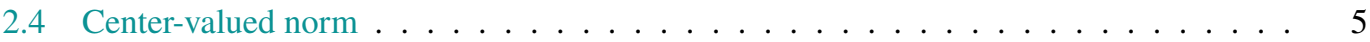

3 Lattice isomorphisms of projection lattices $\quad 6$

4 Ring isomorphisms of locally measurable operator algebras $\quad 14$

5 Questions $r$

\section{Introduction}

Since the very first work by Murray and von Neumann more than 80 years ago [17], the geometry of projections has played the central role in understanding the structure of von Neumann algebras (rings of operators). For a von Neumann algebra $M$, let $\mathcal{P}(M)$ denote the projection lattice of $M$, that is, $\mathcal{P}(M):=\left\{p \in M \mid p=p^{*}=p^{2}\right\}$. In this article, I would like to consider the following question: What is the general form of lattice isomorphisms between projection lattices of von Neumann algebras?

There are several important results related to this question. Let us first think about finite dimensional factors. The case $M=N=\mathbb{M}_{n}(\mathbb{C})$ for $n=1,2$ is not interesting at all. Indeed, if $n=1$, then $\mathcal{P}\left(\mathbb{M}_{n}(\mathbb{C})\right)$ is $\{0,1\}$, and a lattice automorphism of it is the identity mapping. If $n=2$, then a bijection $\Phi$ on $\mathcal{P}\left(\mathbb{M}_{n}(\mathbb{C})\right)$ is a lattice automorphism if and only if $\Phi(0)=0$ and $\Phi(1)=1$. If $M=N=\mathbb{M}_{n}(\mathbb{C})$ for $3 \leq n<\infty$, then the fundamental theorem of projective geometry gives an answer to my question.

(C) The Author(s), 2020. Published by Cambridge University Press. This is an Open Access article, distributed under the terms of the Creative Commons Attribution licence (http://creativecommons.org/licenses/by/4.0/), which permits unrestricted re-use, distribution, and reproduction in any medium, provided the original work is properly cited. 
Recall that a function $f: X \rightarrow Y$ between complex vector spaces is said to be semilinear if it is additive and there exists a ring homomorphism $\sigma: \mathbb{C} \rightarrow \mathbb{C}$ satisfying $f(c x)=\sigma(c) f(x)$ for all $c \in \mathbb{C}$ and $x \in X$.

Theorem 1.1 (Fundamental theorem of projective geometry). Let $3 \leq n<\infty$. Suppose that $\Phi: \mathcal{P}\left(\mathbb{M}_{n}(\mathbb{C})\right) \rightarrow \mathcal{P}\left(\mathbb{M}_{n}(\mathbb{C})\right)$ is a lattice isomorphism. Then there exists a semilinear bijection $f: \mathbb{C}^{n} \rightarrow \mathbb{C}^{n}$ such that $\Phi\left(p_{\xi}\right)=p_{f(\xi)}$ for every $\xi \in \mathbb{C}^{n}$, where $p_{\xi}$ denotes the projection from $\mathbb{C}^{n}$ onto $\mathbb{C} \xi$ for a vector $\xi \in \mathbb{C}^{n}$.

In the case of type $\mathrm{I}_{\infty}$ factors, we can make use of a result from [5]. Recall that a projection $p \in \mathcal{P}(B(H))$ can be identified with its range $p H$, which is a closed subspace of $H$.

Theorem 1.2 ([5, Theorem 1]). Let $X$ and $Y$ be infinite-dimensional complex normed spaces. Let $\mathcal{C}(X)$ (resp. $\mathrm{C}(Y)$ ) denote the lattice of all closed subspaces of $X$ (resp. $Y$ ), ordered by inclusion. Suppose that $\Phi: \mathcal{C}(X) \rightarrow \mathcal{C}(Y)$ is a lattice isomorphism. Then there exists a bicontinuous linear or conjugate-linear bijection $f: X \rightarrow Y$ such that $\Phi(C)=f(C)$ for any $C \in \mathcal{C}(X)$.

See also the classical result [10, Theorem 1], based on orthocomplementation on the lattice $\mathcal{P}(B(H))$.

For type I factors, we may observe a correspondence between lattices and rings. Let $H$ be a Hilbert space with $\operatorname{dim} H \geq 3$. For any lattice automorphism $\Phi: \mathcal{P}(B(H)) \rightarrow \mathcal{P}(B(H))$, take a mapping $f: H \rightarrow H$ as above. It is a semilinear bijection if $\operatorname{dim} H<\infty$, and a linear or conjugate-linear bounded bijection if $\operatorname{dim} H=\infty$. Hence we may construct a ring automorphism $\Psi: B(H) \rightarrow B(H)$ such that $\Phi(l(x))=l(\Psi(x))$ for every $x \in B(H)$ (namely, $\Psi(x):=f \circ x \circ f^{-1}$ ), where $l(x)$ denotes the left support projection of $x$. It is easy to see that the converse also holds. That is, any ring automorphism $\Psi: B(H) \rightarrow B(H)$ determines a lattice automorphism $\Phi$ of $\mathcal{P}(B(H))$ such that $\Phi(l(x))=l(\Psi(x))$ for every $x \in B(H)$.

I next consider finite von Neumann algebras. In the 1930s, motivated by the geometry of projection lattices of type $\mathrm{II}_{1}$ factors, von Neumann produced the beautiful theory on the correspondence between complemented modular lattices and regular rings. One of his achievements [22, Part II, Theorem 4.2], applied to the case of arbitrary type $\mathrm{II}_{1}$ von Neumann algebras, reads as follows:

Theorem 1.3 (von Neumann). Let $M$ and $N$ be von Neumann algebras of type $I_{1}$. Suppose that $\Phi: \mathcal{P}(M) \rightarrow \mathcal{P}(N)$ is a lattice isomorphism. Then there exists a unique ring isomorphism $\Psi: S(M) \rightarrow$ $S(N)$ between the algebras of measurable operators such that $\Phi(l(x))=l(\Psi(x))$ for any $x \in S(M)$.

See Section 2 for the definition of undefined terms and Section 5 for further details about von Neumann's theory.

In the general setting of von Neumann algebras, with an additional assumption, Dye obtained the following result in 1955:

Theorem 1.4 ([3, Corollary of Theorem 1]; see also [4, Theorem 1]). Let $M$ and $N$ be von Neumann algebras without type $I_{2}$ direct summands. Suppose that $\Phi: \mathcal{P}(M) \rightarrow \mathcal{P}(N)$ is a lattice isomorphism with

$$
p q=0 \Longleftrightarrow \Phi(p) \Phi(q)=0
$$

for any $p, q \in \mathcal{P}(M)$. Then there exists a real ${ }^{*}$-isomorphism $\Psi: M \rightarrow N$ that extends $\Phi$.

Each of these results implies that lattice isomorphisms between projection lattices are closely related to ring isomorphisms. See also McAsey's survey [14], which discusses projection lattice isomorphisms in various settings. It is natural to imagine that we can obtain a similar result for arbitrary lattice isomorphisms in the general setting of von Neumann algebras. The main theorem of this article realises it:

Theorem A. Let $M$ and $N$ be two von Neumann algebras. Suppose that $M$ does not admit type $I_{1}$ nor $I_{2}$ direct summands, and that $\Phi: \mathcal{P}(M) \rightarrow \mathcal{P}(N)$ is a lattice isomorphism. Then there exists a unique ring isomorphism $\Psi: L S(M) \rightarrow L S(N)$ such that $\Phi(l(x))=l(\Psi(x))$ for all $x \in L S(M)$. 
Here, $L S(M)$ and $L S(N)$ mean the algebras of locally measurable operators of $M$ and $N$, respectively (see Section 2.2). We remark that the converse of Theorem A can be verified without difficulty. Namely, any ring isomorphism $\Psi: L S(M) \rightarrow L S(N)$ determines a unique lattice isomorphism $\Phi: \mathcal{P}(M) \rightarrow$ $\mathcal{P}(N)$ such that $\Phi(l(x))=l(\Psi(x))$ for all $x \in L S(M)$ (Proposition 3.1). Therefore, Theorem A naturally gives rise to the following:

Question. Let $M, N$ be von Neumann algebras. What is the general form of ring isomorphisms from $L S(M)$ onto $L S(N)$ ?

We can answer this question for type I von Neumann algebras using ring isomorphisms of their centers (Proposition 4.2). Moreover, we obtain the following:

Theorem B. Let $M, N$ be von Neumann algebras of type $I_{\infty}$ or III. If $\Psi: L S(M) \rightarrow L S(N)$ is a ring isomorphism, then there exist a real ${ }^{*}$-isomorphism $\psi: M \rightarrow N$ (which extends to a real ${ }^{*}$-isomorphism from $L S(M)$ onto $L S(N))$ and an invertible element $y \in L S(N)$ such that $\Psi(x)=y \psi(x) y^{-1}, x \in L S(M)$.

I leave the case of type II von Neumann algebras as an open question.

In Section 2, I introduce some tools that I will use later. Section 3 is devoted to the proof of Theorem A. The proof is based on the combination of von Neumann's strategy in [22, Part II, Chapter IV] and a binary relation on the projection lattice which we call LS-orthogonality. After that I give a proof of Dye's theorem as an application of Theorem A. I consider the Question in Section 4 and prove Theorem B. The article ends with a comparison of my result and von Neumann's theory, and several suggestions of further research directions (Section 5).

\section{Preliminaries}

Let $M \subset B(H)$ be a von Neumann algebra. We use the symbol $\sim$ to mean the Murray-von Neumann equivalence relation on $\mathcal{P}(M)$. That is, for $p, q \in \mathcal{P}(M), p \sim q$ means that there exists a partial isometry $v \in M$ such that $p=v v^{*}$ and $q=v^{*} v$. As usual, for $p, q \in \mathcal{P}(M), p \perp q$ means that $p$ and $q$ are orthogonal. That is, $p q=q p=0$, or equivalently, $p H \perp q H$ in the Hilbert space $H$. We use the symbol $p^{\perp}:=1-p$ for $p \in \mathcal{P}(M)$. The symbol $\mathcal{Z}(M)=\{x \in M \mid x y=y x$ for all $y \in M\}$ means the center of $M$.

For $n \in \mathbb{N}=\{1,2, \ldots\}$, we say that $M$ has order $n$ if there exists a collection $p_{1}, \ldots, p_{n}$ of mutually orthogonal projections in $M$ such that $p_{1} \sim p_{2} \sim \cdots \sim p_{n}$ and $\sum_{k=1}^{n} p_{k}=1$. It is well known that every von Neumann algebra without finite type I direct summands has order $n$ for any $n \in \mathbb{N}[9$, Lemma 6.5.6]. In particular, such an algebra has order 3. It follows that every von Neumann algebra $M$ without type $\mathrm{I}_{1}$ and $\mathrm{I}_{2}$ direct summands can be decomposed into the $\left(\ell^{\infty}\right.$-)direct sum of von Neumann algebras $M_{n}, 3 \leq n<\infty$, such that $M_{n}$ has order $n$ for every $n$. If $M$ has order $n \in \mathbb{N}$, then it can be identified with the algebra $\mathbb{M}_{n}(\hat{M})$ of $n \times n$ matrices with entries in some von Neumann algebra $\hat{M}$.

\subsection{Various isomorphisms of von Neumann algebras}

For *-algebras $A$ and $B$, a (not necessarily linear) bijection $\psi: A \rightarrow B$ is called

- a semigroup isomorphism if it is multiplicative,

o a ring isomorphism if it is additive and multiplicative,

- a real algebra isomorphism if it is a real-linear ring isomorphism,

- an algebra isomorphism if it is a complex-linear ring isomorphism,

- a real ${ }^{*}$-isomorphism if it is a real algebra isomorphism and satisfies $\psi\left(x^{*}\right)=\psi(x)^{*}$ for any $x \in A$,

$\circ \mathrm{a}{ }^{*}$-isomorphism if it is a complex-linear real *-isomorphism and

○ a conjugate-linear ${ }^{*}$-isomorphism if it is a conjugate-linear real *-isomorphism.

Lemma 2.1. Let $M$ and $N$ be von Neumann algebras. Suppose that $\psi: M \rightarrow N$ is a bijection.

1. If $M$ is without type $I_{1}$ direct summands and $\psi$ is a semigroup isomorphism, then $\psi$ is a ring isomorphism. 
2. If $M$ does not admit a finite-dimensional ideal and $\psi$ is a ring isomorphism, then $\psi$ is a real algebra isomorphism.

3. If $\psi$ is a real algebra isomorphism, then there exist a real ${ }^{*}$-isomorphism $\psi_{0}: M \rightarrow N$ and an invertible element $y \in N$ such that $\psi(x)=y \psi_{0}(x) y^{-1}$ for any $x \in M$.

4. If $\psi$ is a real ${ }^{*}$-isomorphism, then there exist central projections $p \in M, q \in N, a^{*}$-isomorphism $\psi_{1}: M p \rightarrow N q$ and a conjugate-linear ${ }^{*}$-isomorphism $\psi_{2}: M p^{\perp} \rightarrow N q^{\perp}$ such that $\psi(x)=$ $\psi_{1}(x p)+\psi_{2}\left(x p^{\perp}\right)$ for any $x \in M$.

Proof. Each item is easily obtained from known results.

(1) We can take a projection $p \in \mathcal{P}(M)$ such that both of the central supports of $p$ and $1-p$ are equal to 1. It is easy to see that the following hold: (a) If $x \in M$ satisfies $x M=\{0\}$, then $x=0$; (b) if $x \in M$ satisfies $p M x=\{0\}$, then $x=0$; (c) if $x \in M$ satisfies $p x p M p^{\perp}=\{0\}$, then $p x p=0$. Hence we can apply Martindale's theorem [13, Theorem] to obtain the desired conclusion.

(2) is a consequence of Kaplansky's result [11, Theorem].

I prove (3) and (4) at the same time. Let $\psi: M \rightarrow N$ be a real algebra isomorphism. We know that $\psi(i)^{2}=\psi\left(i^{2}\right)=\psi(-1)=-1$ and that $\psi(i)$ is central in $N$. It follows that $\psi(i)=q i-q^{\perp} i$ for some central projection $q$ of $N$. Set $p:=\psi^{-1}(q)$, which is a central projection of $M$. If $\psi$ is a real *-isomorphism, then $\psi$ restricted to $M p$ is a *-isomorphism from $M p$ onto $N q$, and $\psi$ restricted to $M p^{\perp}$ is a conjugate-linear *-isomorphism from $M p^{\perp}$ onto $N q^{\perp}$; hence the proof of (4) is complete. If $\psi$ is merely a real algebra isomorphism, then $\psi$ restricted to $M p$ is an algebra isomorphism from $M p$ onto $N q$, and $\psi$ restricted to $M p^{\perp}$ determines an algebra isomorphism from $M p^{\perp}$ onto $\overline{N q^{\perp}}$, where $\overline{N q^{\perp}}$ means the complex conjugation of the von Neumann algebra $N q^{\perp}$. For the definition of complex conjugation of von Neumann algebras, see, for example, [19, Section 2.3]. Lastly, we can use the result on the general form of algebra isomorphisms between von Neumann algebras [18, Theorem I] (see also [6] and [20, Section 4.1]) to obtain the desired conclusion.

\subsection{The algebra of locally measurable operators}

Let $M \subset B(H)$ be a von Neumann algebra. In this article, the algebra $L S(M)$ of locally measurable operators with respect to $M$, which I briefly describe in the following, plays a crucial role.

A densely defined closed operator $x$ on $H$ is said to be affiliated with $M$ (and we write $x \eta M$ ) if $y x \subset x y$ for any $y \in M^{\prime}$, where $M^{\prime}:=\{y \in B(H) \mid a y=y a$ for any $a \in M\}$ denotes the commutant of $M$. An operator $x \eta M$ is said to be measurable with respect to $M$ if the spectral projection $\chi_{(c, \infty)}(|x|) \in \mathcal{P}(M)$ is a finite projection in $M$ for some real number $c>0$. An operator $x \eta M$ is said to be locally measurable with respect to $M$ if there exists an increasing sequence $\left\{p_{n}\right\}_{n \geq 1}$ of central projections in $M$ such that $p_{n} \nearrow 1$ and $x p_{n}$ is measurable with respect to $M$ for any $n$. We write $S(M)$ (resp. $L S(M)$ ) to mean the collection of all measurable (resp. locally measurable) operators with respect to $M$. If $x, y \in S(M)$ (resp. $L S(M)$ ), then $x^{*}$ and the closures of $x y, x+y$ are in $S(M)$ (resp. $L S(M)$ ). Using this fact, we can consider $S(M)$ and $L S(M)$ as *-algebras that contain $M$. In what follows, I abbreviate the symbol of the closure of an unbounded operator unless it is confusing. I remark that $L S(M)=M$ holds if and only if $M$ is the direct sum of finite number of type I and III factors. I also remark that if $M$ is finite, then $L S(M)=S(M)$ is the collection of all affiliated operators. See [21, 23] for more details of (locally) measurable operators.

In [16, Lemma 2.2], the following result was obtained:

Lemma 2.2. Let $M$ be a von Neumann algebra and $a \in M_{+}$. Then the following two conditions are equivalent:

1. The element $a$ is invertible in the algebra $L S(M)$.

2. For any $b \in M_{+} \backslash\{0\}$, there exists an $x \in M_{+} \backslash\{0\}$ such that $x \leq a$ and $x \leq b$.

For $x \in L S(M)$, let $l(x) \in \mathcal{P}(M)$ denote the left support of $x$. That is, $l(x):=\bigwedge\{p \in \mathcal{P}(M) \mid p x=x\}$. Similarly, we write $r(x):=\bigwedge\{p \in \mathcal{P}(M) \mid x=x p\}$. Then $l(x)=\chi_{(0, \infty)}\left(\left|x^{*}\right|\right)$ and $r(x)=\chi_{(0, \infty)}(|x|)$ hold. I remark that, for $x, y \in L S(M)$, we have $x y=0$ if and only if $r(x) l(y)=0$. Indeed, if $r(x) l(y)=0$, 
then $x y=x r(x) l(y) y=0$. If $x y=0$, then we have $|x|\left|y^{*}\right|=0$, which implies $\chi_{(\varepsilon, \infty)}(|x|) \chi(\varepsilon, \infty)\left(\left|y^{*}\right|\right)=0$ for every $\varepsilon>0$. Take the limit $\varepsilon \rightarrow 0$ in the strong operator topology to obtain $r(x) l(y)=0$.

\subsection{Halmos's two-projection theorem}

In order to play with projection lattices, it is useful to look at the relative position of a pair of projections. For that, we make use of Halmos's two-projection theorem [8] from the viewpoint of von Neumann algebra theory. Here I recapitulate the argument in [15, Lemma 2.2].

Let $M \subset B(H)$ be a von Neumann algebra and $p, q \in \mathcal{P}(M)$. Set

$$
e_{1}=p-p \wedge q-p \wedge q^{\perp}, \quad e_{2}=p^{\perp}-p^{\perp} \wedge q-p^{\perp} \wedge q^{\perp}
$$

and $x:=e_{1}\left(q-p \wedge q-p^{\perp} \wedge q\right) e_{2}$. By an elementary calculation, we see that $l(x)=e_{1}$ and $r(x)=e_{2}$. By polar decomposition, we can take a partial isometry $v=v_{p, q} \in M$ such that $x=v|x|=\left|x^{*}\right| v, v v^{*}=e_{1}$ and $v^{*} v=e_{2}$.

We can identify each $y \in\left(e_{1}+e_{2}\right) M\left(e_{1}+e_{2}\right)$ with $\left(\begin{array}{cc}e_{1} y e_{1} & e_{1} y v^{*} \\ v y e_{1} & v y v^{*}\end{array}\right) \in \mathbb{M}_{2}\left(e_{1} M e_{1}\right)$. Then $q-p \wedge q-$ $p^{\perp} \wedge q\left(\leq e_{1}+e_{2}\right)$ is identified with

$$
\begin{aligned}
& \left(\begin{array}{cc}
e_{1}\left(q-p \wedge q-p^{\perp} \wedge q\right) e_{1} & e_{1}\left(q-p \wedge q-p^{\perp} \wedge q\right) v^{*} \\
v\left(q-p \wedge q-p^{\perp} \wedge q\right) e_{1} & v\left(q-p \wedge q-p^{\perp} \wedge q\right) v^{*}
\end{array}\right) \\
= & \left(\begin{array}{cc}
e_{1}\left(q-p \wedge q-p^{\perp} \wedge q\right) e_{1} & \left|x^{*}\right| \\
\left|x^{*}\right| & v\left(q-p \wedge q-p^{\perp} \wedge q\right) v^{*}
\end{array}\right) \in \mathbb{M}_{2}\left(e_{1} M e_{1}\right) .
\end{aligned}
$$

Set $a:=\left(e_{1}\left(q-p \wedge q-p^{\perp} \wedge q\right) e_{1}\right)^{1 / 2}$ and $b:=\left(v\left(q-p \wedge q-p^{\perp} \wedge q\right) v^{*}\right)^{1 / 2}$, which are positive injective operators in $M_{p, q}:=e_{1} M e_{1}$. Since $\left(\begin{array}{cc}a^{2} & \left|x^{*}\right| \\ \left|x^{*}\right| & b^{2}\end{array}\right)$ is a projection, some calculations show that $a, b$ and $\left|x^{*}\right|$ commute, $a^{2}+b^{2}=e_{1}$ and $\left|x^{*}\right|=a b$. Thus $q-p \wedge q-p^{\perp} \wedge q$ corresponds to $\left(\begin{array}{ll}a^{2} & a b \\ b a & b^{2}\end{array}\right)$.

Therefore, we can decompose $p$ and $q$ in the following manner:

$$
p=1 \oplus 0 \oplus 1 \oplus 0 \oplus\left(\begin{array}{ll}
1 & 0 \\
0 & 0
\end{array}\right), \quad q=0 \oplus 1 \oplus 1 \oplus 0 \oplus\left(\begin{array}{ll}
a^{2} & a b \\
a b & b^{2}
\end{array}\right),
$$

where $H$ is decomposed as $H=\left(p \wedge q^{\perp}\right) H \oplus\left(p^{\perp} \wedge q\right) H \oplus(p \wedge q) H \oplus\left(p^{\perp} \wedge q^{\perp}\right) H \oplus\left(e_{1}+e_{2}\right) H$ and $a$ and $b$ are positive injective operators in $M_{p, q}\left(=e_{1} M e_{1}\right)$ such that $a^{2}+b^{2}=1_{M_{p, q}}$.

\subsection{Center-valued norm}

Let $M$ be a von Neumann algebra of type I or III and $x \in L S(M)$. Then there exists a unique minimal element $\|\mid x\| \| \in L S(Z(M))_{+}(\subset L S(M))$ with $|x| \leq\|x\| \|$. The mapping $\|\cdot \mid\|: L S(M) \rightarrow L S(Z(M))_{+}$ is called the center-valued norm. Remark that if $M$ is a factor, then $Z(M)$ can be identified with $\mathbb{C}$ and we have $\|x\|=\|x\| \in \mathbb{R}$ for every $x \in M$. Be cautious of the fact that we cannot take such a mapping for a type II von Neumann algebra; that is why we will need to exclude type II cases in the proof of Theorem B.

As is expected, the center-valued norm possesses the following properties: For any $x, y \in L S(M)$ and $a \in L S(Z(M))$, we have (i) $\|x\|=0 \Longrightarrow x=0$; (ii) $\|x+y\| \leq\|x\|+\|y\|$; (iii) $\|a\| \|=|a|$; (iv) $\|a x|\|=|a|\|||x| \mid$; and (v) $\| x y\|\| \leq\|x\|\|\||\|\mid\|$. See [1, Section 2] and references therein for further information about the center-valued norm. 


\section{Lattice isomorphisms of projection lattices}

Part of this section heavily depends on von Neumann's argument in [22, Part II, Chapter IV]. The aim of this section is to give a proof of Theorem A. But first, we consider the converse of Theorem A.

Proposition 3.1. Let $M$ and $N$ be von Neumann algebras. Suppose that $\Psi: L S(M) \rightarrow L S(N)$ is a ring isomorphism. Then there exists a unique lattice isomorphism $\Phi: \mathcal{P}(M) \rightarrow \mathcal{P}(N)$ such that $\Phi(l(x))=l(\Psi(x))$ for any $x \in L S(M)$.

Proof. It is easy to see that $\Psi(0)=0$. Let $x, y \in L S(M)$ satisfy $l(x) \leq l(y)$. Then we have $\{z \in L S(M) \mid$ $z x \neq 0\} \subset\{z \in L S(M) \mid z y \neq 0\}$ and hence $\{z \in L S(N) \mid z \Psi(x) \neq 0\} \subset\{z \in L S(N) \mid z \Psi(y) \neq 0\}$, which in turn leads to $l(\Psi(x)) \leq l(\Psi(y))$. We obtain $l(x) \leq l(y) \Longleftrightarrow l(\Psi(x)) \leq l(\Psi(y))$ for any $x, y \in L S(M)$. Therefore, the mapping $\Phi: \mathcal{P}(M) \rightarrow \mathcal{P}(N)$ defined by $\Phi(p)=l(\Psi(p)), p \in \mathcal{P}(M)$ satisfies the desired condition.

Remark 3.2. The same proof is valid even if we replace a ring isomorphism with a semigroup isomorphism. However, Martindale's result [13] implies that a semigroup isomorphism $\Psi: L S(M) \rightarrow L S(N)$ is automatically a ring isomorphism if $M$ is without type $\mathrm{I}_{1}$ direct summands.

To begin the proof of Theorem A, let us first check the uniqueness of $\Psi$.

Lemma 3.3. Let $M$ be a von Neumann algebra without type $I_{1}$ direct summands. For any $x \in M$, there exists a subset $F \subset M$ with $\# F \leq 9, \sum_{y \in F} y=x$ and the following property: For any $y \in F$, there exists a pair $p, q \in \mathcal{P}(M)$ of mutually orthogonal projections such that $p \sim q$ and either $p y p=y$ or $p y q=y$.

Proof. It suffices to consider the case where $M$ has fixed order $2 \leq n<\infty$. Then we can identify $M$ with $\mathbb{M}_{n}(\hat{M})$ for some von Neumann algebra $\hat{M}$. We can write $x \in M$ as $x=\left(x_{i j}\right)_{1 \leq i, j \leq n} \in \mathbb{M}_{n}(\hat{M})$. It is easy to see that we can take integers $n_{0}:=0 \leq n_{1} \leq n_{2} \leq n=: n_{3}$ such that $n_{1}, n_{2}-n_{1}, n_{3}-n_{2} \leq n / 2$. For $1 \leq k, l \leq 3$, define $x^{k l}=\left(x_{i j}^{k l}\right)_{1 \leq i, j \leq n} \in \mathbb{M}_{n}(\hat{M})$ by $x_{i j}^{k l}=x_{i j}$ if $n_{k-1}+1 \leq i \leq n_{k}$ and $n_{l-1}+1 \leq j \leq n_{l}$, and $x_{i j}^{k l}=0$ otherwise. (Here we are decomposing $x$ into $3 \times 3$ blocks.) Then the nine operators $x^{k l}$, $1 \leq k, l \leq 3$ (some of which may be 0 ), satisfy the desired condition.

Lemma 3.4. Let $M$ be a von Neumann algebra without type $I_{1}$ direct summands. Suppose that $\Psi: L S(M) \rightarrow L S(M)$ is a ring isomorphism with $l(\Psi(x))=l(x)$ for all $x \in L S(M)$. Then $\Psi$ is the identity mapping on $L S(M)$.

Proof. Let $p \in \mathcal{P}(M)$. I prove $\Psi(p)=p$. Since $p p^{\perp}=0$, we have $\Psi(p) \Psi\left(p^{\perp}\right)=0$, which implies $0=r(\Psi(p)) l\left(\Psi\left(p^{\perp}\right)\right)=r(\Psi(p)) p^{\perp}$. We obtain $r(\Psi(p)) \leq p$. We also have the equation $\Psi(p)^{2}=$ $\Psi\left(p^{2}\right)=\Psi(p)$. Hence, we obtain $(p-\Psi(p)) \Psi(p)=0$, which implies $0=(p-\Psi(p)) l(\Psi(p))=$ $(p-\Psi(p)) p$ and $p-\Psi(p)=0$.

In what follows, let $p, q \in \mathcal{P}(M)$ be mutually orthogonal mutually Murray-von Neumann equivalent projections. I next prove that $\Psi(x)=x$ if $x \in M(\subset L S(M))$ satisfies $p x q=x$. By additivity, we may assume $\|x\| \leq 1 / 2$. Then there exists a projection $e \in \mathcal{P}(M)$ such that $e \leq p+q$, peq $=x$. Indeed, let $x=v|x|=\left|x^{*}\right| v$ be the polar decomposition. Take an operator $a \in(p M p)_{+}$such that $\|a\| \leq \pi / 4$ and $\left|x^{*}\right|=\sin a \cos a=(\sin 2 a) / 2$. Then

$$
e:=\cos ^{2} a+v^{*}(\sin a \cos a)+(\sin a \cos a) v+v^{*}\left(\sin ^{2} a\right) v
$$

satisfies this property. We obtain $\Psi(x)=\Psi(p e q)=\Psi(p) \Psi(e) \Psi(q)=p e q=x$.

Suppose that $x \in M$ satisfies $p x p=x$. Take a partial isometry $v \in M$ such that $v v^{*}=p$ and $v^{*} v=q$. Then we have $p(x v) q=x v$ and $q v^{*} p=v^{*}$, and hence $\Psi(x)=\Psi\left(x v v^{*}\right)=\Psi(x v) \Psi\left(v^{*}\right)=x v v^{*}=x$. 
By the additivity of $\Psi$ and Lemma 3.3, we see that $\Psi$ fixes every element in $M$. Let $x \in L S(M)$ and let $x=v|x|$ be its polar decomposition. It is clear that $\Psi(1)=1$. Since $v,(|x|+1)^{-1} \in M$, we obtain

$$
\begin{aligned}
\Psi(x)=\Psi(v|x|) & =\Psi(v) \Psi(|x|) \\
& =v(\Psi(|x|+1)-1)=v\left(\Psi\left((|x|+1)^{-1}\right)^{-1}-1\right) \\
& =v((|x|+1)-1)=v|x|=x
\end{aligned}
$$

Hence we obtain the uniqueness of $\Psi$ in Theorem A. Indeed, if two ring isomorphisms $\Psi, \Psi^{\prime}: L S(M) \rightarrow L S(N)$ satisfy $l(\Psi(x))=l\left(\Psi^{\prime}(x)\right)$ for all $x \in L S(M)$, then we have $l\left(\Psi^{-1} \circ \Psi^{\prime}(x)\right)=$ $l(x)$ for all $x \in L S(M)$, and hence Lemma 3.4 implies $\Psi^{-1} \circ \Psi^{\prime}(x)=x$ for all $x \in L S(M)$.

I introduce a binary relation on $\mathcal{P}(M)$, which is a key to the proof of Theorem A. Let $p, q \in \mathcal{P}(M)$ be two projections with $p \wedge q=0$. By Subsection 2.3, we decompose $p$ and $q$ :

$$
p=1 \oplus 0 \oplus 0 \oplus\left(\begin{array}{ll}
1 & 0 \\
0 & 0
\end{array}\right), \quad q=0 \oplus 1 \oplus 0 \oplus\left(\begin{array}{ll}
a^{2} & a b \\
a b & b^{2}
\end{array}\right) .
$$

We say that $p$ is $L S$-orthogonal to $q$ if the operator $b \in M_{p, q}$ is invertible in $L S\left(M_{p, q}\right)$.

Lemma 3.5. Let $M$ be a von Neumann algebra and $p, q \in \mathcal{P}(M)$. Suppose that $p$ is LS-orthogonal to $q$. Then there exists an invertible element $S=S_{p, q} \in L S(M)$ such that $S(p \vee q)^{\perp}=(p \vee q)^{\perp} S=(p \vee q)^{\perp}$, $S p=p$ and $l\left(S q S^{-1}\right)=p \vee q-p$.

Proof. Set $S:=1 \oplus 1 \oplus 1 \oplus\left(\begin{array}{cc}1 & -a b^{-1} \\ 0 & b^{-1}\end{array}\right)$ with respect to the decomposition as before. Then $S$ is an element in $L S(M)$ with inverse $S^{-1}=1 \oplus 1 \oplus 1 \oplus\left(\begin{array}{ll}1 & a \\ 0 & b\end{array}\right)$. It is easy to see that

$$
S(p \vee q)^{\perp}=(p \vee q)^{\perp} S=(p \vee q)^{\perp}=0 \oplus 0 \oplus 1 \oplus\left(\begin{array}{ll}
0 & 0 \\
0 & 0
\end{array}\right) .
$$

We also have

$$
S p=1 \oplus 0 \oplus 0 \oplus\left(\begin{array}{ll}
1 & 0 \\
0 & 0
\end{array}\right)=p
$$

and

$$
l\left(S q S^{-1}\right)=l\left(0 \oplus 1 \oplus 0 \oplus\left(\begin{array}{ll}
0 & 0 \\
a & 1
\end{array}\right)\right)=0 \oplus 1 \oplus 0 \oplus\left(\begin{array}{ll}
0 & 0 \\
0 & 1
\end{array}\right)=p \vee q-p
$$

Lemma 3.6. Let $M$ be a von Neumann algebra and $p, q \in \mathcal{P}(M)$ be two projections with $p \wedge q=0$. Then the following are equivalent:

1. The projection $p$ is LS-orthogonal to $q$.

2. There exists a lattice automorphism $\Phi$ of $\mathcal{P}(M)$ such that $\Phi(p) \perp \Phi(q)$.

3. If a projection $p_{0} \in \mathcal{P}(M)$ satisfies $p_{0} \leq p$ and $p_{0} \vee q=p \vee q$, then $p_{0}=p$.

4. The projection $q$ is LS-orthogonal to $p$.

Proof. (1) $\Rightarrow(2)$ Take $S \in L S(M)$ as in Lemma 3.5 and let $\Phi$ be the unique lattice isomorphism such that $\Phi(l(x))=l\left(S x S^{-1}\right), x \in L S(X)$.

(2) $\Rightarrow(3)$ is clear.

(3) $\Rightarrow$ (1) We use the decomposition (3.1). By Lemma 2.2, if (1) does not hold, then there exists an element $d \in M_{p, q^{+}} \backslash\{0\}$ such that $\left\{x \in M_{p, q+} \mid x \leq b, x \leq d\right\}=\{0\}$. Take the nonzero spectral 
projection $p_{1}:=\chi(\|d\| / 2,\|d\|](d) \in \mathcal{P}\left(M_{p, q}\right)$. It follows that

$$
\left\{x \in M_{p, q+} \mid x \leq b, x \leq p_{1}\right\}=\{0\} .
$$

Indeed, if $x \in M_{p, q+}$ satisfies $x \leq b$ and $x \leq p_{1}$, take a positive real number $c$ with $c \leq 1$ and $c \leq\|d\| / 2$ and then $c x \leq c b \leq b$ and $c x \leq c p_{1} \leq d$; hence, $c x=0$ and we obtain $x=0$. Set $p_{0}:=1 \oplus 0 \oplus 0 \oplus\left(\begin{array}{cr}1-p_{1} & 0 \\ 0 & 0\end{array}\right) \in \mathcal{P}(M)$. Then $p_{0} \leq p$ and $p_{0} \neq p$. I prove that $p_{0} \vee q=p \vee q$, or equivalently, $\left(\begin{array}{cc}1-p_{1} & 0 \\ 0 & 0\end{array}\right) \vee\left(\begin{array}{ll}a^{2} & a b \\ a b & b^{2}\end{array}\right)=1_{\mathbb{M}_{2}\left(M_{p, q}\right)}$, which is in turn equivalent to

$$
\left(\begin{array}{cc}
p_{1} & 0 \\
0 & 1
\end{array}\right) \wedge\left(\begin{array}{cc}
b^{2} & -a b \\
-a b & a^{2}
\end{array}\right)=0_{\mathbb{M}_{2}\left(M_{p, q}\right)} .
$$

We have

$$
\begin{aligned}
& \left(\begin{array}{ll}
1 & 0 \\
0 & 0
\end{array}\right)\left(\left(\begin{array}{cc}
p_{1} & 0 \\
0 & 1
\end{array}\right) \wedge\left(\begin{array}{cc}
b^{2} & -a b \\
-a b & a^{2}
\end{array}\right)\right)\left(\begin{array}{ll}
1 & 0 \\
0 & 0
\end{array}\right) \\
& \leq\left(\begin{array}{ll}
1 & 0 \\
0 & 0
\end{array}\right)\left(\begin{array}{cc}
p_{1} & 0 \\
0 & 1
\end{array}\right)\left(\begin{array}{ll}
1 & 0 \\
0 & 0
\end{array}\right)=\left(\begin{array}{cc}
p_{1} & 0 \\
0 & 0
\end{array}\right)
\end{aligned}
$$

and

$$
\begin{aligned}
& \left(\begin{array}{ll}
1 & 0 \\
0 & 0
\end{array}\right)\left(\left(\begin{array}{cc}
p_{1} & 0 \\
0 & 1
\end{array}\right) \wedge\left(\begin{array}{cc}
b^{2} & -a b \\
-a b & a^{2}
\end{array}\right)\right)\left(\begin{array}{ll}
1 & 0 \\
0 & 0
\end{array}\right) \\
& \leq\left(\begin{array}{ll}
1 & 0 \\
0 & 0
\end{array}\right)\left(\begin{array}{cc}
b^{2} & -a b \\
-a b & a^{2}
\end{array}\right)\left(\begin{array}{ll}
1 & 0 \\
0 & 0
\end{array}\right)=\left(\begin{array}{cc}
b^{2} & 0 \\
0 & 0
\end{array}\right) .
\end{aligned}
$$

Since the square-root mapping preserves the order of positive operators, (3.2) implies that the square root of the operator

$$
\left(\begin{array}{ll}
1 & 0 \\
0 & 0
\end{array}\right)\left(\left(\begin{array}{cc}
p_{1} & 0 \\
0 & 1
\end{array}\right) \wedge\left(\begin{array}{cc}
b^{2} & -a b \\
-a b & a^{2}
\end{array}\right)\right)\left(\begin{array}{ll}
1 & 0 \\
0 & 0
\end{array}\right)
$$

is equal to 0 . Hence

$$
\left(\begin{array}{ll}
1 & 0 \\
0 & 0
\end{array}\right)\left(\left(\begin{array}{cc}
p_{1} & 0 \\
0 & 1
\end{array}\right) \wedge\left(\begin{array}{cc}
b^{2} & -a b \\
-a b & a^{2}
\end{array}\right)\right)\left(\begin{array}{ll}
1 & 0 \\
0 & 0
\end{array}\right)=0
$$

or equivalently, $\left(\begin{array}{cc}p_{1} & 0 \\ 0 & 1\end{array}\right) \wedge\left(\begin{array}{cc}b^{2} & -a b \\ -a b & a^{2}\end{array}\right) \leq\left(\begin{array}{ll}0 & 0 \\ 0 & 1\end{array}\right)$ holds. However, we know $\left(\begin{array}{ll}0 & 0 \\ 0 & 1\end{array}\right) \wedge\left(\begin{array}{cc}b^{2} & -a b \\ -a b & a^{2}\end{array}\right)=0$, so we finally obtain (3.3).

Exchanging the roles of $p$ and $q$, we also obtain (2) $\Leftrightarrow$ (4).

Let us recall the setting of Theorem A: 'Let $M, N$ be von Neumann algebras. Suppose that $M$ is without type $\mathrm{I}_{1}$ and $\mathrm{I}_{2}$ direct summands and $\Phi: \mathcal{P}(M) \rightarrow \mathcal{P}(N)$ is a lattice isomorphism.' By Lemma 3.6, we see that $\Phi$ preserves LS-orthogonality in both directions, that is, for any $p, q \in \mathcal{P}(M), p$ and $q$ are LS-orthogonal if and only if $\Phi(p)$ and $\Phi(q)$ are LS-orthogonal. 
In what follows, I show the existence of $\Psi$ as in the statement of Theorem A in the case that $M$ has order 3. Thus $M$ can be identified with $\mathbb{M}_{3}(\hat{M})$ for some von Neumann algebra $\hat{M}$. Set

$$
e_{1}^{M}:=\left(\begin{array}{lll}
1 & 0 & 0 \\
0 & 0 & 0 \\
0 & 0 & 0
\end{array}\right), e_{2}^{M}:=\left(\begin{array}{lll}
0 & 0 & 0 \\
0 & 1 & 0 \\
0 & 0 & 0
\end{array}\right), e_{3}^{M}:=\left(\begin{array}{lll}
0 & 0 & 0 \\
0 & 0 & 0 \\
0 & 0 & 1
\end{array}\right) \in \mathcal{P}\left(\mathbb{M}_{3}(\hat{M})\right)
$$

Set $e_{1}:=\Phi\left(e_{1}^{M}\right), e_{2}:=\Phi\left(e_{2}^{M}\right), e_{3}:=\Phi\left(e_{3}^{M}\right)$. We know that $e_{1} \vee e_{2}$ is LS-orthogonal to $e_{3}$, and $e_{1}$ is LS-orthogonal to $e_{2}$. In addition, we know $e_{1} \vee e_{2} \vee e_{3}=1$. Take $S_{e_{1} \vee e_{2}, e_{3}}$ and $S_{e_{1}, e_{2}}$ as in the statement of Lemma 3.5. Consider the lattice automorphism $\varphi: \mathcal{P}(N) \rightarrow \mathcal{P}(N)$ determined by the condition $\varphi(l(x))=l\left(S_{e_{1}, e_{2}} S_{e_{1} \vee e_{2}, e_{3}} x S_{e_{1} \vee e_{2}, e_{3}}^{-1} S_{e_{1}, e_{2}}^{-1}\right)\left(=l\left(S_{e_{1}, e_{2}} S_{e_{1} \vee e_{2}, e_{3}} x\right)\right), x \in L S(N)$. A moment's calculation shows that $\varphi\left(e_{1}\right), \varphi\left(e_{2}\right), \varphi\left(e_{3}\right)$ are mutually orthogonal and $\varphi\left(e_{1}\right)+\varphi\left(e_{2}\right)+\varphi\left(e_{3}\right)=1_{N}$.

Lemma 3.7. We have $\varphi\left(e_{1}\right) \sim \varphi\left(e_{2}\right) \sim \varphi\left(e_{3}\right)$ in $N$.

Proof. Subsection 2.3 implies that for $p, q \in \mathcal{P}(N)$, if $p \vee q=1$ and $p \wedge q=0$, then $p^{\perp} \sim q$. Since $\varphi \circ \Phi$ is a lattice isomorphism, we obtain

$$
\varphi\left(e_{1}\right)=\varphi \circ \Phi\left(e_{1}^{M}\right) \sim\left(\varphi \circ \Phi\left(\frac{1}{2}\left(\begin{array}{lll}
1 & 1 & 0 \\
1 & 1 & 0 \\
0 & 0 & 2
\end{array}\right)\right)\right)^{\perp} \sim \varphi \circ \Phi\left(e_{2}^{M}\right)=\varphi\left(e_{2}\right) .
$$

Similarly, we obtain $\varphi\left(e_{1}\right) \sim \varphi\left(e_{3}\right)$.

It suffices to consider $\varphi \circ \Phi$ instead of $\Phi$. Hence we can identify $N$ with $\mathbb{M}_{3}(\hat{N})$ for some von Neumann algebra $\hat{N}$, and we can assume $\Phi\left(e_{1}^{M}\right)=e_{1}^{N}, \Phi\left(e_{2}^{M}\right)=e_{2}^{N}$ and $\Phi\left(e_{3}^{M}\right)=e_{3}^{N}$, where

$$
e_{1}^{N}:=\left(\begin{array}{lll}
1 & 0 & 0 \\
0 & 0 & 0 \\
0 & 0 & 0
\end{array}\right), e_{2}^{N}:=\left(\begin{array}{lll}
0 & 0 & 0 \\
0 & 1 & 0 \\
0 & 0 & 0
\end{array}\right), e_{3}^{N}:=\left(\begin{array}{lll}
0 & 0 & 0 \\
0 & 0 & 0 \\
0 & 0 & 1
\end{array}\right) \in \mathcal{P}\left(\mathbb{M}_{3}(\hat{N})\right) .
$$

Let $x \in L S(\hat{M})$. Suppose that $\hat{M} \subset B(K)$. Viewing $x$ as a closed operator, we see that the collection

$$
\left\{\left(\begin{array}{c}
\xi \\
x \xi \\
0
\end{array}\right) \in K \oplus K \oplus K \mid \xi \in \operatorname{dom} x\right\}
$$

is a closed subspace in $K \oplus K \oplus K$. Take the projection $P_{12}[x] \in \mathcal{P}(B(K \oplus K \oplus K))$ onto this subspace. Then we have

$$
P_{12}[x]=\left(\begin{array}{ccc}
\left(1+x^{*} x\right)^{-1} & \left(1+x^{*} x\right)^{-1} x^{*} & 0 \\
x\left(1+x^{*} x\right)^{-1} & x\left(1+x^{*} x\right)^{-1} x^{*} & 0 \\
0 & 0 & 0
\end{array}\right)
$$

and hence $P_{12}[x] \in \mathcal{P}\left(\mathbb{M}_{3}(\hat{M})\right)$. Similarly, let $P_{13}[x], P_{23}[x] \in \mathcal{P}\left(\mathbb{M}_{3}(\hat{M})\right)$, respectively, denote the projections onto

$$
\left\{\left(\begin{array}{c}
\xi \\
0 \\
x \xi
\end{array}\right) \in K \oplus K \oplus K \mid \xi \in \operatorname{dom} x\right\}, \quad\left\{\left(\begin{array}{c}
0 \\
\xi \\
x \xi
\end{array}\right) \in K \oplus K \oplus K \mid \xi \in \operatorname{dom} x\right\} .
$$

Lemma 3.8. Let $Q \in \mathcal{P}\left(\mathbb{M}_{3}(\hat{M})\right)$. Then the following conditions are equivalent:

1. There exists an $x \in L S(\hat{M})$ such that $Q=P_{12}[x]$.

2. $Q \vee e_{2}^{M}=e_{1}^{M} \vee e_{2}^{M}$, and $Q$ is LS-orthogonal to $e_{2}^{M}$. 
Proof. $(1) \Rightarrow(2)$ Let $Q=P_{12}[x]$. Since $\left(1+x^{*} x\right)^{-1}$ is a positive injective operator, we have $Q \vee e_{2}^{M}=$ $e_{1}^{M} \vee e_{2}^{M}$ by (3.4). Let $x=v|x|$ be the polar decomposition. By (3.4), we have

$$
Q=P_{12}[x]=\left(\begin{array}{ccc}
\left(1+|x|^{2}\right)^{-1} & \left(1+|x|^{2}\right)^{-1}|x| v^{*} & 0 \\
v|x|\left(1+|x|^{2}\right)^{-1} & v|x|\left(1+|x|^{2}\right)^{-1}|x| v^{*} & 0 \\
0 & 0 & 0
\end{array}\right)
$$

Hence we have

$$
Q \wedge e_{2}^{M} \leq\left(\begin{array}{lcl}
0 & 0 & 0 \\
0 & v|x|\left(1+|x|^{2}\right)^{-1}|x| v^{*} & 0 \\
0 & 0 & 0
\end{array}\right)
$$

Since $1-v|x|\left(1+|x|^{2}\right)^{-1}|x| v^{*}$ is a positive injective operator, we see that $Q \wedge e_{2}^{M}=0$. As in (3.1), we can decompose $e_{2}^{M}$ and $Q$ in the following form:

$$
e_{2}^{M}=1 \oplus 0 \oplus 0 \oplus\left(\begin{array}{ll}
1 & 0 \\
0 & 0
\end{array}\right), Q=0 \oplus 1 \oplus 0 \oplus\left(\begin{array}{ll}
a^{2} & a b \\
a b & b^{2}
\end{array}\right) .
$$

We also have

$$
e_{1}^{M}=0 \oplus 1 \oplus 0 \oplus\left(\begin{array}{ll}
0 & 0 \\
0 & 1
\end{array}\right)
$$

with respect to the same decomposition. Recall that $\left(1+x^{*} x\right)^{-1}$ is invertible in $L S(\hat{M})$, or equivalently, $e_{1}^{M} Q e_{1}^{M}$ is invertible in $\operatorname{LS}\left(e_{1}^{M} M e_{1}^{M}\right)$. This means that

$$
0 \oplus 1 \oplus 0 \oplus\left(\begin{array}{cc}
0 & 0 \\
0 & b^{2}
\end{array}\right)
$$

is invertible in $L S\left(e_{1}^{M} M e_{1}^{M}\right)$, which in particular implies the invertibility of $b$ in $L S\left(M_{e_{2}^{M}, Q}\right)$.

(2) $\Rightarrow$ (1) As in (3.1), we can decompose $e_{2}^{M}$ and $Q$ in the following form:

$$
e_{2}^{M}=1 \oplus 0 \oplus 0 \oplus\left(\begin{array}{ll}
1 & 0 \\
0 & 0
\end{array}\right), Q=0 \oplus 1 \oplus 0 \oplus\left(\begin{array}{ll}
a^{2} & a b \\
a b & b^{2}
\end{array}\right) .
$$

Note that $b$ is invertible as a locally measurable operator. It follows that

$$
e_{1}^{M}=0 \oplus 1 \oplus 0 \oplus\left(\begin{array}{ll}
0 & 0 \\
0 & 1
\end{array}\right)
$$

Consider the partial isometry

$$
w=0 \oplus 1 \oplus 0 \oplus\left(\begin{array}{ll}
0 & 0 \\
a & b
\end{array}\right) .
$$

We have $w w^{*}=e_{1}^{M}$ and $w^{*} w=Q$. Moreover, $e_{1}^{M} w e_{1}^{M}$ is a positive invertible element in $L S\left(e_{1}^{M} M e_{1}^{M}\right)$. Thus a moment's reflection shows that there exist $w_{1}, w_{2} \in \hat{M}$ such that $w_{1} \geq 0, w_{1}$ is invertible in $L S(\hat{M})$ and $w=\left(\begin{array}{ccc}w_{1} & w_{2} & 0 \\ 0 & 0 & 0 \\ 0 & 0 & 0\end{array}\right) \in \mathbb{M}_{3}(\hat{M})$. (Here $w_{1}$ corresponds to $e_{1}^{M} w e_{1}^{M}$.) Set $x=w_{2}^{*} w_{1}^{-1}$. Since $w w^{*}=e_{1}^{M}$, we obtain $w_{1}^{2}+w_{2} w_{2}^{*}=1_{\hat{M}}$. Hence,

$$
1+x^{*} x=1+w_{1}^{-1} w_{2} w_{2}^{*} w_{1}^{-1}=1+w_{1}^{-1}\left(1-w_{1}^{2}\right) w_{1}^{-1}=w_{1}^{-2} .
$$


It follows by (3.4) that

$$
P_{12}[x]=\left(\begin{array}{ccc}
\left(1+x^{*} x\right)^{-1} & \left(1+x^{*} x\right)^{-1} x^{*} & 0 \\
x\left(1+x^{*} x\right)^{-1} & x\left(1+x^{*} x\right)^{-1} x^{*} & 0 \\
0 & 0 & 0
\end{array}\right)=\left(\begin{array}{ccc}
w_{1}^{2} & w_{1} w_{2} & 0 \\
w_{2}^{*} w_{1} & w_{2}^{*} w_{2} & 0 \\
0 & 0 & 0
\end{array}\right)=w^{*} w=Q .
$$

Corollary 3.9. Let $k \in\{12,13,23\}$. There exists a bijection $\psi_{k}: \operatorname{LS}(\hat{M}) \rightarrow \operatorname{LS}(\hat{N})$ such that $\Phi\left(P_{k}[x]\right)=P_{k}\left[\psi_{k}(x)\right]$. Moreover, $x \in L S(\hat{M})$ is invertible in $L S(\hat{M})$ if and only if $\psi_{k}(x)$ is invertible in $\operatorname{LS}(\hat{N})$

Proof. Since $\Phi$ is a lattice isomorphism with $\Phi\left(e_{1}^{M}\right)=e_{1}^{N}$ and $\Phi\left(e_{2}^{M}\right)=e_{2}^{N}$, the first half of the case $k=12$ follows from Lemma 3.8. For $x \in L S(\hat{M})$, let $P_{21}[x] \in \mathcal{P}\left(\mathbb{M}_{3}(\hat{M})\right)$ denote the projection onto

$$
\left\{\left(\begin{array}{c}
x \xi \\
\xi \\
0
\end{array}\right) \in K \oplus K \oplus K \mid \xi \in \operatorname{dom} x\right\}
$$

thus,

$$
P_{21}[x]=\left(\begin{array}{ccc}
x\left(1+x^{*} x\right)^{-1} x^{*} & x\left(1+x^{*} x\right)^{-1} & 0 \\
\left(1+x^{*} x\right)^{-1} x^{*} & \left(1+x^{*} x\right)^{-1} & 0 \\
0 & 0 & 0
\end{array}\right)
$$

It is easy to see that for $x, y \in L S(\hat{M})$, the equation $P_{12}[x]=P_{21}[y]$ holds if and only if $x$ is invertible in $L S(\hat{M})$ and $y=x^{-1}$. Therefore, Lemma 3.8 implies that an operator $x \in L S(\hat{M})$ is invertible in $L S(\hat{M})$ if and only if $P_{12}[x]$ is LS-orthogonal to $e_{1}^{M}$ and $P_{12}[x] \vee e_{1}^{M}=e_{1}^{M} \vee e_{2}^{M}$. Thus $\psi_{12}$ preserves invertibility. The other cases can be shown similarly.

In particular, the operators $\psi_{12}(1), \psi_{13}(1)$ are invertible in $L S(\hat{N})$. Consider the lattice automorphism $\phi$ of $\mathcal{P}\left(\mathbb{M}_{3}(\hat{N})\right)$ determined by $\phi(l(x))=l\left(S x S^{-1}\right)$, where $S=\left(\begin{array}{ccc}1 & 0 & 0 \\ 0 & \psi_{12}(1)^{-1} & 0 \\ 0 & 0 & \psi_{13}(1)^{-1}\end{array}\right)$. We see that $\phi\left(e_{i}^{N}\right)=e_{i}^{N}, i=1,2,3$, and

$$
\phi \circ \Phi\left(P_{12}\left[1_{\hat{M}}\right]\right)=P_{12}\left[1_{\hat{N}}\right], \phi \circ \Phi\left(P_{13}\left[1_{\hat{M}}\right]\right)=P_{13}\left[1_{\hat{N}}\right] .
$$

Considering $\phi \circ \Phi$ instead of $\Phi$, we can assume $\Phi\left(P_{12}\left[1_{\hat{M}}\right]\right)=P_{12}\left[1_{\hat{N}}\right]$ and $\Phi\left(P_{13}\left[1_{\hat{M}}\right]\right)=P_{13}\left[1_{\hat{N}}\right]$, or equivalently, $\psi_{12}(1)=\psi_{13}(1)=1$.

Lemma 3.10. For any $x, y \in L S(\hat{M})$, we have

$$
P_{13}[x y]=\left(P_{23}[-x] \vee P_{12}[y]\right) \wedge\left(e_{1}^{M} \vee e_{3}^{M}\right) .
$$

Proof. Let $\hat{M} \subset B(K)$. We know that the range of $P_{23}[-x] \vee P_{12}[y]$ is the closure of

$$
V:=\left\{\left(\begin{array}{c}
\eta \\
\xi+y \eta \\
-x \xi
\end{array}\right) \in K \oplus K \oplus K \mid \xi \in \operatorname{dom} x, \eta \in \operatorname{dom} y\right\} .
$$

In particular, we have $\left(\begin{array}{c}\eta \\ 0 \\ x y \eta\end{array}\right) \in V$ for any $\eta \in \operatorname{dom} y$ with $y \eta \in \operatorname{dom} x$. Since the collection $\{\eta \in \operatorname{dom} y \mid$ $y \eta \in \operatorname{dom} x\}$ is a core of the operator $x y \in L S(\hat{M})$, we have $P_{13}[x y] \leq\left(P_{23}[-x] \vee P_{12}[y]\right) \wedge\left(e_{1}^{M} \vee e_{3}^{M}\right)$. 
We claim that the orthogonal complement $V^{\perp}$ of $V$ is

$$
\left\{\left(\begin{array}{c}
-y^{*} x^{*} \zeta \\
x^{*} \zeta \\
\zeta
\end{array}\right) \in K \oplus K \oplus K \mid \zeta \in \operatorname{dom} x^{*}, x^{*} \zeta \in \operatorname{dom} y^{*}\right\} .
$$

It is clear that any $\left(\begin{array}{c}-y^{*} x^{*} \zeta \\ x^{*} \zeta \\ \zeta\end{array}\right)$ is an element in $V^{\perp}$. If $\left(\begin{array}{c}\zeta_{1} \\ \zeta_{2} \\ \zeta_{3}\end{array}\right) \in V^{\perp}$, then

$$
0=\left\langle\left(\begin{array}{c}
\zeta_{1} \\
\zeta_{2} \\
\zeta_{3}
\end{array}\right),\left(\begin{array}{c}
0 \\
\xi \\
-x \xi
\end{array}\right)\right\rangle=\left\langle\zeta_{2}, \xi\right\rangle-\left\langle\zeta_{3}, x \xi\right\rangle
$$

for any $\xi \in \operatorname{dom} x$, and hence we obtain $\zeta_{3} \in \operatorname{dom} x^{*}, \zeta_{2}=x^{*} \zeta_{3}$. By the equation

$$
0=\left\langle\left(\begin{array}{c}
\zeta_{1} \\
\zeta_{2} \\
\zeta_{3}
\end{array}\right),\left(\begin{array}{c}
\eta \\
y \eta \\
0
\end{array}\right)\right\rangle
$$

for $\eta \in \operatorname{dom} y$, we obtain the claim. Let $\left(\begin{array}{l}h_{1} \\ h_{2} \\ h_{3}\end{array}\right)$ belong to the range of $\left(P_{23}[-x] \vee P_{12}[y]\right) \wedge\left(e_{1}^{M} \vee e_{3}^{M}\right)$, which is equal to the orthogonal complement of $V^{\perp} \cup\left\{\left(\begin{array}{l}0 \\ k \\ 0\end{array}\right) \in K \oplus K \oplus K \mid k \in K\right\}$. Then we have $h_{2}=0$ and

$$
0=\left\langle\left(\begin{array}{c}
h_{1} \\
0 \\
h_{3}
\end{array}\right),\left(\begin{array}{c}
-y^{*} x^{*} \zeta \\
x^{*} \zeta \\
\zeta
\end{array}\right)\right)=-\left\langle h_{1}, y^{*} x^{*} \zeta\right\rangle+\left\langle h_{3}, \zeta\right\rangle
$$

for any $\zeta \in \operatorname{dom} x^{*}$ with $x^{*} \zeta \in \operatorname{dom} y^{*}$. We know that $\left\{\zeta \in \operatorname{dom} x^{*} \mid x^{*} \zeta \in \operatorname{dom} y^{*}\right\}$ is a core of the operator $y^{*} x^{*} \in L S(\hat{M})$. Thus we obtain $h_{1} \in \operatorname{dom}\left(y^{*} x^{*}\right)^{*}=\operatorname{dom}(x y)$ and $h_{3}=(x y) h_{1}$ (here we view $x y$ as a closed operator in $L S(\hat{M}))$.

Lemma 3.11. We have $\psi_{12}=\psi_{13}=\psi_{23}=: \psi$. Moreover, $\psi: L S(\hat{M}) \rightarrow \operatorname{LS}(\hat{N})$ is multiplicative.

Proof. Let $x, y \in L S(\hat{M})$. By Lemma 3.10, we have

$$
P_{13}[x y]=\left(P_{23}[-x] \vee P_{12}[y]\right) \wedge\left(e_{1}^{M} \vee e_{3}^{M}\right)
$$

and hence

$$
\begin{aligned}
P_{13}\left[\psi_{13}(x y)\right]=\Phi\left(P_{13}[x y]\right) & =\Phi\left(\left(P_{23}[-x] \vee P_{12}[y]\right) \wedge\left(e_{1}^{M} \vee e_{3}^{M}\right)\right) \\
& =\left(\Phi\left(P_{23}[-x]\right) \vee \Phi\left(P_{12}[y]\right)\right) \wedge\left(\Phi\left(e_{1}^{M}\right) \vee \Phi\left(e_{3}^{M}\right)\right) \\
& =\left(P_{23}\left[\psi_{23}(-x)\right] \vee P_{12}\left[\psi_{12}(y)\right]\right) \wedge\left(e_{1}^{N} \vee e_{3}^{N}\right)
\end{aligned}
$$

It follows by Lemma 3.10 again (applied to $N$ instead of $M$ ) that

$$
\left(P_{23}\left[\psi_{23}(-x)\right] \vee P_{12}\left[\psi_{12}(y)\right]\right) \wedge\left(e_{1}^{N} \vee e_{3}^{N}\right)=P_{13}\left[-\psi_{23}(-x) \psi_{12}(y)\right] .
$$

Thus we obtain $P_{13}\left[-\psi_{23}(-x) \psi_{12}(y)\right]=P_{13}\left[\psi_{13}(x y)\right]$, which implies $-\psi_{23}(-x) \psi_{12}(y)=\psi_{13}(x y)$. 
In particular, setting $x=y=1$, we obtain $\psi_{23}(-1)=-1$. Setting $x=1$, we obtain $-\psi_{23}(-1) \psi_{12}(y)=$ $\psi_{13}(y)$, hence $\psi_{12}(y)=\psi_{13}(y)$. Moreover, setting $y=1$, we obtain $-\psi_{23}(-x) \psi_{12}(1)=\psi_{13}(x)$, hence $-\psi_{23}(-x)=\psi_{13}(x)$. Thus $\psi_{12}(x) \psi_{12}(y)=-\psi_{23}(-x) \psi_{12}(y)=\psi_{13}(x y)=\psi_{12}(x y)$. Therefore, $\psi_{12}$ is multiplicative. It follows that $\psi_{12}(-1)$ is central in $L S(\hat{N}), \psi_{12}(-1)^{2}=1$ and $\psi_{12}(-1) y \neq y$ for any $y \neq 0$, and hence we obtain $\psi_{12}(-1)=-1$. We reach the equation $\psi_{13}=\psi_{12}=\psi_{23}$.

Lemma 3.12. The mapping $\psi$ is additive.

Proof. Let $x, y \in L S(\hat{M})$. Consider the projections

$$
f=\left(P_{12}[x] \vee e_{3}^{M}\right) \wedge\left(P_{13}[1] \vee e_{2}^{M}\right) \quad \text { and } \quad g=\left(P_{12}[y] \vee P_{13}[1]\right) \wedge\left(e_{2}^{M} \vee e_{3}^{M}\right)
$$

By an argument similar to that in the proof of Lemma 3.10, we can check the following: The range of $f$ is equal to

$$
\left\{\left(\begin{array}{c}
\xi \\
x \xi \\
\xi
\end{array}\right) \in K \oplus K \oplus K \mid \xi \in \operatorname{dom} x\right\}
$$

and the range of $g$ is equal to

$$
\left\{\left(\begin{array}{c}
0 \\
-y \eta \\
\eta
\end{array}\right) \in K \oplus K \oplus K \mid \eta \in \operatorname{dom} y\right\}
$$

hence $(f \vee g) \wedge\left(e_{1}^{M} \vee e_{2}^{M}\right)=P_{12}[x+y]$. Apply $\Phi$ to both sides to obtain the desired conclusion.

Define a mapping $\Psi: L S\left(\mathbb{M}_{3}(\hat{M})\right) \rightarrow L S\left(\mathbb{M}_{3}(\hat{N})\right)$ by $\Psi\left(\left(x_{i j}\right)_{i j}\right):=\left(\psi\left(x_{i j}\right)\right)_{i j}, x_{i j} \in L S(\hat{M})$, $i, j=1,2,3$. The preceding lemmas imply that $\Psi$ is a ring isomorphism from $\operatorname{LS}\left(\mathbb{M}_{3}(\hat{M})\right)$ onto $L S\left(\mathbb{M}_{3}(\hat{N})\right)$.

Lemma 3.13. We have $\Phi(l(x))=l(\Psi(x))$ for any $x \in L S\left(\mathbb{M}_{3}(\hat{M})\right)$.

Proof. I partly imitate Dye's argument in the proof of [3, Lemma 7]. By Lemma 3.4, it suffices to show that the lattice isomorphism $\Phi^{\prime}: \mathcal{P}\left(\mathbb{M}_{3}(\hat{M})\right) \rightarrow \mathcal{P}\left(\mathbb{M}_{3}(\hat{N})\right)$ determined by $l(\Psi(x))=\Phi^{\prime}(l(x))$, $x \in L S\left(\mathbb{M}_{3}(\hat{M})\right)$, satisfies $\Phi=\Phi^{\prime}$. For $x \in L S(\hat{M})$, we have

$$
\begin{aligned}
\Phi\left(P_{12}[x]\right)=P_{12}[\psi(x)] & =l\left(\begin{array}{ccc}
1 & 0 & 0 \\
\psi(x) & 0 & 0 \\
0 & 0 & 0
\end{array}\right) \\
& =l\left(\Psi\left(\begin{array}{lll}
1 & 0 & 0 \\
x & 0 & 0 \\
0 & 0 & 0
\end{array}\right)\right)=\Phi^{\prime}\left(l\left(\begin{array}{lll}
1 & 0 & 0 \\
x & 0 & 0 \\
0 & 0 & 0
\end{array}\right)\right)=\Phi^{\prime}\left(P_{12}[x]\right) .
\end{aligned}
$$

Similarly, we see that $\Phi(p)=\Phi^{\prime}(p)$ for any $p \in\left\{P_{k}[x] \mid x \in L S(\hat{M}), k=12,23,13\right\}$.

Let $x_{2}, x_{3} \in L S(\hat{M})$. Consider the projection $P_{x_{2}, x_{3}} \in \mathcal{P}\left(\mathbb{M}_{3}(\hat{M})\right)$ onto the closed subspace

$$
\left\{\left(\begin{array}{c}
\xi \\
x_{2} \xi \\
x_{3} \xi
\end{array}\right) \in K \oplus K \oplus K \mid \xi \in \operatorname{dom} x_{2} \cap \operatorname{dom} x_{3}\right\} .
$$

It is not difficult to see that this projection is equal to $\left(P_{12}\left[x_{2}\right] \vee e_{3}^{M}\right) \wedge\left(P_{13}\left[x_{3}\right] \vee e_{2}^{M}\right)$. It follows that $\Phi\left(P_{x_{2}, x_{3}}\right)=\Phi^{\prime}\left(P_{x_{2}, x_{3}}\right)$. 
Consider an arbitrary nonzero projection $p=\left(p_{i, j}\right)_{1 \leq i, j \leq 3} \in \mathcal{P}\left(\mathbb{M}_{3}(\hat{M})\right)$. By Zorn's lemma, to show that $\Phi(p)=\Phi^{\prime}(p)$, it suffices to find a nonzero subprojection $(p \geq) q \in \mathcal{P}\left(\mathbb{M}_{3}(\hat{M})\right)$ such that $\Phi(q)=\Phi^{\prime}(q)$. Note that $p_{i i}=\sum_{1 \leq k \leq 3} p_{i k} p_{i k}^{*}$, hence we see that $p_{i i} \neq 0$ for some $i \in\{1,2,3\}$.

If $p_{11} \neq 0$, set $e:=\chi_{\left(\left\|p_{11}\right\| / 2,\left\|p_{11}\right\|\right]}\left(p_{11}\right) \in \mathcal{P}(\hat{M}) \backslash\{0\}$ and $x_{1}:=p_{11}^{-1} e \in \hat{M}$. It follows that the projection $q \in \mathcal{P}\left(\mathbb{M}_{3}(\hat{M})\right)$ onto the subspace

$$
\left\{\left(\begin{array}{c}
p_{11} \xi \\
p_{21} \xi \\
p_{31} \xi
\end{array}\right) \in K \oplus K \oplus K \mid \xi \in e K\right\}=\left\{\left(\begin{array}{c}
\eta \\
p_{21} x_{1} \eta \\
p_{31} x_{1} \eta
\end{array}\right) \in K \oplus K \oplus K \mid \eta \in e K\right\}
$$

is a nonzero subprojection of $p$. Since $q=P_{p_{21} x_{1}, p_{31} x_{1}} \wedge\left(\left(P_{12}\left[e^{\perp}\right] \wedge e_{1}^{M}\right) \vee e_{2}^{M} \vee e_{3}^{M}\right)$, we obtain $\Phi(q)=\Phi^{\prime}(q)$.

If $p_{11}=0$ and $p_{22} \neq 0$, we have $p \leq e_{2}^{M} \vee e_{3}^{M}$. Then a similar discussion applies. If $p_{11}=p_{22}=0$, then $p_{33} \in \mathcal{P}(\hat{M})$. Use the equation $\left(P_{13}[1] \vee P_{13}\left[p_{33}^{\perp}\right]\right) \wedge e_{3}^{M}=p$, which can be verified easily, to obtain the desired conclusion.

Therefore, the proof of Theorem A is complete in the case where $M$ has order 3. The same discussion with a slight modification is valid in any case where $M$ has order $n$ with $3 \leq n<\infty$. We know that a projection lattice isomorphism preserves central projections because a projection $p$ in a von Neumann algebra $M$ is central if and only if $\{q \in \mathcal{P}(M) \mid p \vee q=1, p \wedge q=0\}=\left\{p^{\perp}\right\}$. Since every von Neumann algebra without type $\mathrm{I}_{1}$ and $\mathrm{I}_{2}$ direct summands decomposes into the direct sum of algebras of order $3 \leq n<\infty$, now it easy to complete the proof of Theorem A in the general case.

In what follows, I give a proof of Theorem 1.4 by Dye (in the case the von Neumann algebras are without commutative direct summands) as an application of Theorem A. The following proof is partly based on Feldman's argument [4, Proof of Theorem 3].

Let $M$ and $N$ be von Neumann algebras without type $\mathrm{I}_{1}$ and $\mathrm{I}_{2}$ direct summands, and suppose that $\Phi: \mathcal{P}(M) \rightarrow \mathcal{P}(N)$ is a lattice isomorphism. Suppose further that we have $p q=0$ if and only if $\Phi(p) \Phi(q)=0$ for any pair $p, q \in \mathcal{P}(M)$. By Theorem A, there exists a unique ring isomorphism $\Psi: L S(M) \rightarrow L S(N)$ such that $\Phi(l(x))=l(\Psi(x))$ for any $x \in L S(M)$.

Then we have $\Psi(p)=\Phi(p) \in \mathcal{P}(N)$ for every $p \in \mathcal{P}(M)$. Indeed, since $p^{2}=p$ and $p p^{\perp}=0$, we have $\Psi(p)^{2}=\Psi(p)$ and $\Psi(p) \Psi\left(p^{\perp}\right)=0$. Thus we have $r(\Psi(p)) l\left(\Psi\left(p^{\perp}\right)\right)=0$. The assumption implies $l\left(\Psi\left(p^{\perp}\right)\right)=\Phi\left(p^{\perp}\right)=\Phi(p)^{\perp}=l(\Psi(p))^{\perp}$, and thus we obtain $r(\Psi(p)) \leq l(\Psi(p))$. By the equation $(l(\Psi(p))-\Psi(p)) \Psi(p)=0$, we obtain $0=(l(\Psi(p))-\Psi(p)) l(\Psi(p))=l(\Psi(p))-\Psi(p)$. Hence $\Psi(p)=l(\Psi(p))=\Phi(p) \in \mathcal{P}(N)$.

Consider the ring automorphism $x \mapsto \Psi^{-1}\left(\Psi\left(x^{*}\right)^{*}\right)$ of $L S(M)$. This fixes every projection, hence Lemma 3.4 implies that $x=\Psi^{-1}\left(\Psi\left(x^{*}\right)^{*}\right)$, or equivalently, $\Psi(x)^{*}=\Psi\left(x^{*}\right)$ for each $x \in L S(M)$. It follows that $\Psi$ maps the self-adjoint part of $L S(M)$ onto that of $L S(N)$. Since $\Psi$ preserves squares, $\Psi$ restricted to self-adjoint parts preserves order in both directions. Since $\Psi(1)=1, \Psi$ restricts to a real ${ }^{*}$-isomorphism from $M$ onto $N$ and extends $\Phi$, which is the desired conclusion.

\section{Ring isomorphisms of locally measurable operator algebras}

By Section 3, lattice isomorphisms between projection lattices are in one-to-one correspondence with ring isomorphisms between the algebras of locally measurable operators. Hence the following question is well motivated:

Question. Let $M, N$ be von Neumann algebras. What is the general form of ring isomorphisms from $L S(M)$ onto $L S(N)$ ? 
Lemma 4.1. Let $M, N$ be general von Neumann algebras. Let

$$
\begin{aligned}
& M=\left(\bigoplus_{n \geq 1} M_{\mathrm{I}_{n}}\right) \oplus M_{\mathrm{I}_{\infty}} \oplus M_{\mathrm{II}_{1}} \oplus M_{\mathrm{II}_{\infty}} \oplus M_{\mathrm{III}}, \\
& N=\left(\bigoplus_{n \geq 1} N_{\mathrm{I}_{n}}\right) \oplus N_{\mathrm{I}_{\infty}} \oplus N_{\mathrm{II}_{1}} \oplus N_{\mathrm{II}_{\infty}} \oplus N_{\mathrm{III}}
\end{aligned}
$$

be the type decompositions, where $M_{j}, N_{j}$ are von Neumann algebras of type $j$. Suppose that $\Psi: L S(M) \rightarrow L S(N)$ is a ring isomorphism. Then there exist ring isomorphisms $\psi_{j}: L S\left(M_{j}\right) \rightarrow$ $L S\left(N_{j}\right)$ such that $\Psi(x)=\psi_{j}(x)$ for any $x \in L S\left(M_{j}\right)(\subset L S(M))$.

Proof. It is easy to see that $\Psi$ maps the collection of central projections in $M$ onto that in $N$. Hence it suffices to show that if $M, N$ are of type $j, k \in\left\{\mathrm{I}_{n} \mid n \geq 1\right\} \cup\left\{\mathrm{I}_{\infty}, \mathrm{II}_{1}, \mathrm{II}_{\infty}, \mathrm{III}\right\}$, respectively, then $j=k$. Consider the lattice isomorphism $\Phi: \mathcal{P}(M) \rightarrow \mathcal{P}(N)$ as in Proposition 3.1. It is easy to see that a projection $p \in \mathcal{P}(M)$ is abelian (namely, $p M p$ is an abelian von Neumann algebra) if and only if $\Phi(p)$ is abelian. Moreover, a projection $p \in \mathcal{P}(M)$ is finite if and only if $\Phi(p) \in \mathcal{P}(N)$ is finite. Indeed, if $p$ is not finite, then there exist mutually orthogonal nonzero subprojections $p_{1}, p_{2}, p_{3}$ of $p$ such that $p_{1} \sim p_{2} \sim p_{3} \sim p_{1}+p_{2}$. The same argument as in the proof of Lemma 3.7 implies $\Phi\left(p_{1}\right) \sim \Phi\left(p_{3}\right) \sim \Phi\left(p_{1}+p_{2}\right)$, which shows that $\Phi(p)$ is not finite. Similarly, if $\Phi(p)$ is not finite, then $p$ is not finite. The rest of the proof is a standard argument of von Neumann algebra theory, and I omit the details (see, e.g., [9, Chapter 6]).

Therefore, the Question reduces to the case where both $M$ and $N$ are of type $j, j \in\left\{\mathrm{I}_{n} \mid n \geq 1\right\}$ $\cup\left\{\mathrm{I}_{\infty}, \mathrm{II}_{1}, \mathrm{II}_{\infty}, \mathrm{III}\right\}$.

First consider the Question in the case where $M, N$ are von Neumann algebras of type $\mathrm{I}_{n}$. Suppose that $L S(M)$ is ring isomorphic to $L S(N)$. Since the central projection lattices of $M$ and $N$ are lattice isomorphic, we see that the center of $M$ is *-isomorphic to that of $N$. Hence there exists a commutative von Neumann algebra $A$ such that $M \cong N \cong \mathbb{M}_{n}(A)$. Therefore, it suffices to think about ring automorphisms of $L S\left(\mathbb{M}_{n}(A)\right)$, which can be identified with the collection of all $n \times n$ matrices with entries in $L S(A)$. Note that $A$ can be identified with the algebra $L^{\infty}(\mu)$ of all complex-valued essentially bounded measurable functions (modulo almost-everywhere equivalence) for some measure $\mu$. Then $L S(A)$ corresponds to $L^{0}(\mu)$, which denotes the collection of all complex-valued measurable functions. Note that any ring automorphism $\psi$ of $L S(A)$ determines a ring automorphism $\psi^{\prime}$ of $L S\left(\mathbb{M}_{n}(A)\right)$ by the formula $\psi^{\prime}\left(\left(x_{i j}\right)\right)=\left(\psi\left(x_{i j}\right)\right)_{i j}$. The following proposition slightly generalizes (but can be shown by exactly the same argument as in) [1, Theorem 3.3].

Proposition 4.2. Let $n \geq 1$ be an integer and $A$ be a commutative von Neumann algebra. Suppose that $\Psi$ is a ring automorphism of $L S\left(\mathbb{M}_{n}(A)\right)$. Then there exist a ring automorphism $\psi: L S(A) \rightarrow L S(A)$ and an invertible element $y$ in $L S\left(\mathbb{M}_{n}(A)\right)$ such that $\Psi(x)=y \psi^{\prime}(x) y^{-1}, x \in L S\left(\mathbb{M}_{n}(A)\right)$.

Proof. Note that $\Psi$ restricts to a ring automorphism $\psi$ of the center of $L S\left(\mathbb{M}_{n}(A)\right)$, which is canonically isomorphic to $L S(A)$. Then $\Psi \circ \psi^{\prime-1}$ fixes every element in the center of $L S\left(\mathbb{M}_{n}(A)\right)$. We can apply [1, Theorem 3.1] to obtain the desired conclusion.

There exist highly nontrivial examples of ring automorphisms of $L S(A)=L^{0}(\mu)$ for a commutative von Neumann algebra $A$. For example, consider the case $A=\mathbb{C}=L S(A)$. There are many ring automorphisms of $\mathbb{C}$ that are far from real-linear. Consider the case where $\mu$ is an atomless measure. It is known $[12,(1) \Leftrightarrow(6)$ of Theorem 3.4] (see also [12, Remark 6.3]) that there exists a (complex-linear) algebra automorphism $\psi$ of $L^{0}(\mu)$ such that $\psi(p)=p$ for any $p \in \mathcal{P}(A)$ but $\psi \neq \operatorname{id}_{L^{0}(\mu)}$. It seems that these examples are beyond the scope of the theory of operator algebras.

In contrast, we can give a purely operator algebraic solution to the Question for type $\mathrm{I}_{\infty}$ or III as Theorem B. This improves on [1, Theorem 3.8], in which algebra isomorphisms of the case of type $\mathrm{I}_{\infty}$ were considered. 
Proof of Theorem B. Beware of the fact that $\Psi$ restricts to a lattice isomorphism between the central projection lattices of $M$ and $N$. I first prove:

Claim There exists an operator $a \in L S(\mathcal{Z}(N))_{+}$such that $\|\Psi(x)\| \leq a$ for any $x \in M(\subset L S(M))$ with $\|x\| \leq 1$.

Assume that this claim does not hold. I will obtain a contradiction in Step 4.

Step 1 I prove that there exists a central projection $e$ in $M$ such that for any $n \geq 1$ there exists some $\overline{x \in M}$ with $\|x\| \leq 1$ and $\|\Psi(x)\| \geq n \Psi(e)$.

Assume for a while that the center $Z(M)$ of $M$ admits a faithful normal state $\tau: Z(M) \rightarrow \mathbb{C}$. For each positive integer $n$, consider the collection

$$
E_{n}:=\{e \in \mathcal{P}(Z(M)) \mid \text { there exists } x \in M \text { with }\|x\| \leq 1 \text { and }\|\Psi(x)\| \geq n \Psi(e)\} .
$$

Suppose that $e, f$ belong to this collection. Take $x, y \in M$ such that $\|x\|,\|y\| \leq 1$ and $\|\Psi(x)\| \| \geq n \Psi(e)$, $\|\Psi \Psi(y)\| \geq n \Psi(f)$. Then the element $x^{\prime}:=x e+y e^{\perp}$ satisfies $\left\|x^{\prime}\right\| \leq 1$ and

$$
\begin{aligned}
\left\|\Psi\left(x^{\prime}\right)\right\| & =\left\|\Psi\left(x e+y e^{\perp}\right)\right\| \\
& =\left\|\Psi(x) \Psi(e)+\Psi(y) \Psi(e)^{\perp}\right\| \\
& =\|\| \Psi(x)\|\Psi(e)+\| \Psi(y) \| \Psi(e)^{\perp} \\
& \geq n \Psi(e)+n \Psi(f) \Psi(e)^{\perp} \\
& =n \Psi(e) \vee \Psi(f)=n \Psi(e \vee f) .
\end{aligned}
$$

Hence we have $e \vee f \in E_{n}$, which implies that $E_{n}$ is upward directed. Set $c_{n}:=\sup \left\{\tau(e) \mid e \in E_{n}\right\}$. We can take an increasing sequence $\left\{e^{(k)}\right\} \subset E_{n}$ such that $\tau\left(e^{(k)}\right) \rightarrow c_{n}$ as $k \rightarrow \infty$. For each $k$, take $x^{(k)} \in M$ such that $\left\|x^{(k)}\right\| \leq 1$ and $\left\|\Psi\left(x^{(k)}\right)\right\| \geq n \Psi\left(e^{(k)}\right)$. Some calculations show that the element

$$
x^{\prime \prime}:=x^{(1)} e^{(1)}+\sum_{k \geq 2} x^{(k)}\left(e^{(k)}-e^{(k-1)}\right) \in M
$$

satisfies $\left\|x^{\prime \prime}\right\| \leq 1$ and $\left\|\Psi\left(x^{\prime \prime}\right)\right\| \geq n \Psi\left(e^{(k)}\right)$ for every $k$. This implies that for the projection $e_{n}:=$ $\bigvee E_{n} \in \mathcal{P}(Z(M))$ there exists $x_{n} \in \mathcal{P}(Z(M))$ such that $\left\|x_{n}\right\| \leq 1$ and $\left\|\Psi\left(x_{n}\right)\right\| \geq n \Psi\left(e_{n}\right)$.

Clearly, $\left\{e_{n}\right\}$ is a decreasing sequence. Assume that $e_{n} \rightarrow 0$ as $n \rightarrow \infty$; then the element $a=$ $\Psi\left(1+\sum_{n \geq 1} e_{n}\right) \in L S(Z(N))_{+}$satisfies the property of the Claim, which contradicts our assumption. Hence we have $e_{n} \rightarrow e \in \mathcal{P}(\mathcal{Z}(M)) \backslash\{0\}$ as $n \rightarrow \infty$, and $e$ satisfies the desired property. Since every von Neumann algebra can be decomposed into the direct sum of von Neumann algebras whose centers admit faithful normal states, the same holds for arbitrary $M$ and $N$.

Considering the restriction of $\Psi$ to a ring isomorphism from $\operatorname{LS}(M e)$ onto $\operatorname{LS}(N \Psi(e))$, we can assume that for any $n \geq 1$ there exists some $x \in M$ with $\|x\| \leq 1$ and $\|\Psi(x)\| \| \geq n$.

Step 2 I prove that for any $a \in L S(Z(N))_{+}$there exists some $x \in M$ with $\|x\| \leq 1$ and $\|\Psi(x)\| \geq a$.

Let $a \in L S(Z(N))_{+}$. We can take a sequence of mutually orthogonal central projections $\left\{f_{n}\right\}$ such that $a \leq \sum_{n \geq 1} n f_{n}$. For each $n$, take $x_{n} \in M$ such that $\left\|x_{n}\right\| \leq 1$ and $\left\|\Psi\left(x_{n}\right)\right\| \geq n f_{n}$. Some calculations show that the element $x:=\sum_{n \geq 1} x_{n} \Psi^{-1}\left(f_{n}\right)$ satisfies $\|x\| \leq 1$ and $\|\Psi(x)\| \geq \sum_{n \geq 1} n f_{n} \geq a$.

Step 3 I prove that for any $p \in \mathcal{P}(M)$ with $p \sim p^{\perp}$ and any $a \in L S(Z(N))_{+}$, there exists an element $\overline{x \in M}$ with $\operatorname{pxp}=x,\|x\| \leq 1$ and $\|\Psi(x)\| \| \geq a$.

Take a partial isometry $v \in M$ such that $v v^{*}=p$ and $v^{*} v=p^{\perp}$. Since $\Psi$ is a ring isomorphism, for any $x \in M$ we have

$$
\begin{aligned}
\Psi(x) & =\Psi\left(p x p+p x p^{\perp}+p^{\perp} x p+p^{\perp} x p^{\perp}\right) \\
& =\Psi(p x p)+\Psi\left(p x v^{*}\right) \Psi(v)+\Psi\left(v^{*}\right) \Psi(v x p)+\Psi\left(v^{*}\right) \Psi\left(v x v^{*}\right) \Psi(v) .
\end{aligned}
$$


For a given $a \in L S(Z(N))_{+}$, set

$$
b:=4 a+4 a\|\| \Psi(v)\left\|\left|+4 a\left\|\left|\Psi ( v ^ { * } ) \left\|\left|+4 a\|\| \Psi(v)\|\|\left\|\Psi\left(v^{*}\right)\right\|\right| \in L S(Z(N))_{+} .\right.\right.\right.\right.\right.
$$

Step 2 implies that there exists $x \in M$ with $\|x\| \leq 1$ and

$$
\begin{aligned}
b & \leq\|\mid \Psi(x)\| \\
& \leq\|\| \Psi(\text { pxp })\|+\| \Psi\left(p x v^{*}\right)\|\|\|\Psi(v)\|+\left\|\Psi\left(v^{*}\right)\right\|\|\| \Psi(v x p)\|+\| \Psi\left(v^{*}\right)\|\|\left\|\Psi\left(v x v^{*}\right)\right\|\|\| \Psi(v) \| .
\end{aligned}
$$

Hence there exists a quadruple $f_{1}, f_{2}, f_{3}, f_{4}$ of central projections in $N$ such that $f_{1}+f_{2}+f_{3}+f_{4}$ $=1,\|\| \Psi($ pxp $)\left\|f_{1} \geq b f_{1} / 4,\right\|\left|\Psi\left(p x v^{*}\right)\|\|\|\mid \Psi(v)\| f_{2} \geq b f_{2} / 4,\left\|\Psi\left(v^{*}\right)\right\|\|\| \Psi(v x p) \| f_{3} \geq b f_{3} / 4\right.$ and $\left\|\left|\Psi\left(v^{*}\right)\left\|\left|\left\|\left|\Psi\left(v x v^{*}\right)\right|\right\|\right|\right\|\right| \Psi(v)\right\| f_{4} \geq b f_{4} / 4$. Set

$$
x^{\prime}:=\operatorname{pxp} \Psi^{-1}\left(f_{1}\right)+p x v^{*} \Psi^{-1}\left(f_{2}\right)+v x p \Psi^{-1}\left(f_{3}\right)+v x v^{*} \Psi^{-1}\left(f_{4}\right) .
$$

Then we have $p x^{\prime} p=x^{\prime},\left\|x^{\prime}\right\| \leq 1$ and

$$
\begin{aligned}
\left\|\Psi\left(x^{\prime}\right)\right\| & =\| \Psi(\text { pxp }) f_{1}+\Psi\left(p x v^{*}\right) f_{2}+\Psi(v x p) f_{3}+\Psi\left(v x v^{*}\right) f_{4} \| \\
& =\| \Psi(\text { pxp })\left\|f_{1}+\right\| \Psi\left(p x v^{*}\right)\left\|f_{2}+\right\| \Psi(v x p)\left\|f_{3}+\right\| \Psi\left(v x v^{*}\right) \| f_{4} \\
& \geq \frac{1}{4} b\left(f_{1}+\|\Psi(v)\|^{-1} f_{2}+\left\|\Psi\left(v^{*}\right)\right\|^{-1} f_{3}+\|\Psi(v)\|^{-1}\left\|\Psi\left(v^{*}\right)\right\|^{-1} f_{4}\right) \geq a .
\end{aligned}
$$

(Note that $\|\Psi(v)\|\|,\| \Psi\left(v^{*}\right)\|\|$ are invertible in $L S(z(N))$.)

Step 4 Since $M$ is properly infinite, we can take a sequence $\left\{p_{n}\right\}_{n \geq 1}$ of mutually orthogonal projections $\overline{\text { in } M}$ such that $p_{n} \sim p_{n}^{\perp}, n \geq 1$. By Step 3, for each $n \geq 1$ we can take an element $x_{n} \in M$ with $p_{n} x_{n} p_{n}=x_{n},\left\|x_{n}\right\| \leq 1$ and $\left\|\Psi\left(x_{n}\right)\right\| \geq n\left\|\Psi\left(p_{n}\right)\right\|$. Set $x:=\sum_{n \geq 1} x_{n} \in M$ (which is well defined, since $p_{n}, n \geq 1$, are mutually orthogonal). For every $n \geq 1$, we have

$$
\left\|\left|\Psi(x)\|\|\left\|\Psi\left(p_{n}\right)\right\|\|\geq\| \Psi(x) \Psi\left(p_{n}\right)\|\mid=\| \Psi\left(x p_{n}\right)\|\|=\left\|\Psi\left(x_{n}\right)\right\| \geq \geq n\left\|\Psi\left(p_{n}\right)\right\| \| .\right.\right.
$$

Since $\left\|\Psi\left(p_{n}\right)\right\|$ is invertible in $L S(Z(N))$, we obtain $\|\Psi(x)\| \| n$ for all $n \in \mathbb{N}$, a contradiction. This completes the proof of the Claim.

Step 5 It follows that there exists an element $a \in L S(Z(N))_{+}$such that $\|\Psi(x)\| \| \leq$ for any $x \in M$ with $\overline{\|x\| \leq}$. By the same discussion applied to $\Psi^{-1}$, we also obtain an element $a^{\prime} \in L S(Z(M))_{+}$such that $\left\|\Psi^{-1}(y)\right\| \leq a^{\prime}$ for any $y \in N$ with $\|y\| \leq 1$. We can take a sequence $e_{n}$ of central projections in $M$ such that $e_{n} \nearrow 1$ and $\Psi$ restricts to a norm-bicontinuous ring isomorphism $\Psi_{n}$ from $M e_{n}$ onto $N \Psi\left(e_{n}\right)$, $n \geq 1$. By Lemma 2.1 we can verify the statement for each $\Psi_{n}$, which suffices to complete the proof.

Corollary 4.3. Let $M, N$ be von Neumann algebras of type $I_{\infty}$ or III. Suppose that $\Phi: \mathcal{P}(M) \rightarrow \mathcal{P}(N)$ is a lattice isomorphism. Then there exist a real ${ }^{*}$-isomorphism $\psi: M \rightarrow N$ and an invertible element $y \in L S(N)$ such that $\Phi(p)=l(y \psi(p)), p \in \mathcal{P}(M)$.

\section{Questions}

I skeptically conjecture that Theorem B also holds for type II von Neumann algebras:

Conjecture 5.1. Let $M$ and $N$ be von Neumann algebras of type II. Suppose that $\Psi: L S(M) \rightarrow L S(N)$ is a ring isomorphism. Then there exist an invertible operator $y \in L S(N)$ and a real ${ }^{*}$-isomorphism $\psi: M \rightarrow N$ such that $\Psi(x)=y \psi(x) y^{-1}$ for any $x \in L S(M)$.

Not much is known about the structure of the algebra $L S(M)$ for a type II (in particular, $\mathrm{II}_{1}$ ) von Neumann algebra $M$. I do not know whether or not such a $\Psi$ is automatically real-linear even in the case where $M$ and $N$ are (say, approximately finite-dimensional) $\mathrm{II}_{1}$ factors. Note that $L S(M)$ cannot 
have a Banach algebra structure because of the fact that an element of $L S(M)$ can have an empty or dense spectral set. Hence it seems difficult to make use of automatic continuity results on algebra isomorphisms as in [2]. However, I suspect that at least the following weaker statement holds:

Conjecture 5.2. Let $M$ and $N$ be von Neumann algebras of type II. If $\mathcal{P}(M)$ and $\mathcal{P}(N)$ are lattice isomorphic, or equivalently, if $L S(M)$ and $L S(N)$ are ring isomorphic, then $M$ and $N$ are real *isomorphic (or equivalently, $M$ and $N$ are Jordan ${ }^{*}$-isomorphic).

In another direction, I compare Theorem A with von Neumann's theory of complemented modular lattices and regular rings. Von Neumann axiomatised projection lattices of type $\mathrm{II}_{1}$ von Neumann algebras and completed the amazing theory on the correspondence between the vast classes of complemented modular lattices and regular rings. Let us briefly recall this theory from [22, Part II].

Definition 5.3. A lattice $L$ with greatest element 1 and least element 0 is complemented if for each $a \in L$ there exists $b \in L$ such that $a \vee b=1, a \wedge b=0$. A lattice $L$ is modular if the equation $(a \vee b) \wedge c=a \vee(b \wedge c)$ holds for any $a, b, c \in L$ with $a \leq c$.

Let $L$ be a complemented modular lattice. Two elements $a, b \in L$ are said to be perspective if there exists $c \in L$ such that $a \vee c=1=b \vee c$ and $a \wedge c=0=b \wedge c$. Let $n$ be a positive integer. We say $L$ has order $n$ if there exist pairwise perspective elements $a_{1}, a_{2}, \ldots, a_{n} \in L$ with $a_{1} \vee a_{2} \vee \cdots \vee a_{n}=1$ and $\left(\bigvee_{i \in I_{1}} a_{i}\right) \wedge\left(\bigvee_{j \in I_{2}} a_{j}\right)=0$ for any disjoint $I_{1}, I_{2} \subset\{1,2, \ldots, n\}$.

Definition 5.4. A (von Neumann) regular ring is a $\operatorname{ring} R$ with unit such that for each $x \in R$ there exists $y \in R$ such that $x y x=x$.

Let $R$ be a regular ring. A right ideal $\mathfrak{a}$ of $R$ is principal if it is generated by one element of $R$.

Let $M$ be a von Neumann algebra. Then $\mathcal{P}(M)$ is a complemented lattice. It is not difficult to show that the following three conditions are equivalent:

$\circ$ The von Neumann algebra $M$ is finite.

- The lattice $\mathcal{P}(M)$ is modular.

- The ring $L S(M)$ is regular.

Theorem 5.5 (von Neumann). If $R$ is a regular ring, then the collection $L$ of all principal right ideals of $R$, ordered by inclusion, forms a complemented modular lattice.

We call $L$ in the statement of this theorem the right ideal lattice of $R$.

Theorem 5.6 (von Neumann). Let $R_{1}, R_{2}$ be regular rings with right ideal lattices $L_{1}, L_{2}$, respectively. Suppose that $L_{1}$ has order $n \geq 3$. If $\Phi: L_{1} \rightarrow L_{2}$ is a lattice isomorphism, then there exists a unique ring isomorphism $\Psi: R_{1} \rightarrow R_{2}$ such that $\Phi(\mathfrak{a})=\Psi(\mathfrak{a}), \mathfrak{a} \in L_{1}$.

Theorem 5.7 (von Neumann). Let $L$ be a complemented modular lattice with order $n \geq 4$. Then there exists a regular ring $R$ such that the right ideal lattice of $R$ is lattice isomorphic to $L$.

Let $M$ be a finite von Neumann algebra and $\mathfrak{a} \subset L S(M)$ a principal right ideal generated by $a \in L S(M)$. It is an easy exercise to show that $\mathfrak{a}=\{x \in L S(M) \mid l(x) \leq l(a)\}$. Hence we obtain an identification of the right ideal lattice of $L S(M)$ with the projection lattice $\mathcal{P}(M)$. In particular, Theorem 1.3 is a corollary of von Neumann's results. See also [7], which deals with the history of the study of regular rings in connection with functional analysis.

Von Neumann's theory, applied to the setting of von Neumann algebras, is valid only for finite von Neumann algebras. In this article, I prove that there exists a complete correspondence between lattice isomorphisms and ring isomorphisms in the general setting of von Neumann algebras. Hence I believe that one might be able to generalize von Neumann's theory to a broader class of lattices that covers projection lattices of any von Neumann algebras (of fixed order $n \geq 3$ or 4 ). This is left as a research programme in the future. 
Acknowledgments. I appreciate Yasuyuki Kawahigashi, who is my advisor, for invaluable support. I am also indebted to Peter Šemrl and Yuhei Suzuki, who encouraged me to begin working on lattice isomorphisms. This work was supported by the Leading Graduate Course for Frontiers of Mathematical Sciences and Physics (FMSP) and the JSPS Research Fellowship for Young Scientists (KAKENHI Grant Number 19J14689), MEXT, Japan. Part of this work was completed during my visit to Gilles Pisier at IMJ-PRG, Paris. That visit was supported by the JSPS Overseas Challenge Program for Young Researchers.

Conflict of Interest: None.

\section{References}

[1] S. Albeverio, S. Ayupov, K. Kudaybergenov and R. Djumamuratov, 'Automorphisms of central extensions of type I von Neumann algebras', Studia Math. 207 (2011) 1-17.

[2] H. G. Dales, Banach Algebras and Automatic Continuity, London Mathematical Society Monographs, New Series, 24 (New York, The Clarendon Press, 2000).

[3] H. A. Dye, 'On the geometry of projections in certain operator algebras', Ann. of Math. (2) 61 (1955) $73-89$.

[4] J. Feldman, 'Isomorphisms of finite type II rings of operators', Ann. of Math. (2) 63 (1956) 565-571.

[5] P. A. Fillmore and W. E. Longstaff, 'On isomorphisms of lattices of closed subspaces', Canad. J. Math. 36 (1984) $820-829$.

[6] L. T. Gardner, 'On isomorphisms of C-algebras', Amer. J. Math. 87 (1965) 384-396.

[7] K. R. Goodearl, 'Von Neumann regular rings: Connections with functional analysis', Bull. Amer. Math. Soc. (N.S.) 4 (1981) 125-134.

[8] P. R. Halmos, ‘Two subspaces', Trans. Amer. Math. Soc. 144 (1969) 381-389.

[9] R. V. Kadison and J. R. Ringrose, Fundamentals of the Theory of Operator Algebras, Vol. II (Orlando, Academic Press, 1986).

[10] S. Kakutani and G. W. Mackey, 'Ring and lattice characterization of complex Hilbert space', Bull. Amer. Math. Soc. 52 (1946) 727-733.

[11] I. Kaplansky, 'Ring isomorphisms of Banach algebras', Canad. J. Math. 6 (1954) 374-381.

[12] A. G. Kusraev, 'Automorphisms and derivations in an extended complex f-algebra', Sibirsk. Mat. Zh. 47 (2006) 97-107; translation in Sib. Math. J. 47 (2006) 77-85.

[13] W. S. MartindaleIII, 'When are multiplicative mappings additive?', Proc. Amer. Math. Soc. 21 (1969) 695-698.

[14] M. McAsey, 'Spatial implementation of lattice isomorphisms: Selfadjoint and nonselfadjoint operator algebras and operator theory', Contemp. Math. 120 (1991) 113-115.

[15] M. Mori, 'Isometries between projection lattices of von Neumann algebras', J. Funct. Anal. 276 (2019) 3511-3528.

[16] M. Mori, 'Order isomorphisms of operator intervals in von Neumann algebras', Integral Equations Operator Theory 91 (2019) 11.

[17] F. J. Murray and J. von Neumann, 'On rings of operators', Ann. of Math. (2) 37 (1936) 116-229.

[18] T. Okayasu, 'A structure theorem of automorphisms of von Neumann algebras', Tohoku Math. J. (2) 20 (1968) $199-206$.

[19] G. Pisier, Tensor Products of $C^{*}$-algebras and Operator Spaces (Cambridge, Cambridge University Press, 2020).

[20] S. Sakai, $C^{*}$-algebras and $W^{*}$-algebras, Ergebnisse der Mathematik und ihrer Grenzgebiete, 60 (Springer, New York, 1971).

[21] I. E. Segal, 'A non-commutative extension of abstract integration', Ann. of Math. (2) 57 (1953) 401-457.

[22] J. von Neumann, Continuous Geometry Princeton Mathematical Series, 25 (Princeton, Princeton University Press, 1960).

[23] F. J. Yeadon, 'Convergence of measurable operators', Math. Proc. Cambridge Philos. Soc. 74 (1973) $257-268$. 